\title{
Crack path tracking from full field measurements: a novel empirical methodology
}

\author{
S. Feld-Payet ${ }^{1} \quad$ | G. Le Besnerais ${ }^{2} \quad$ | V. Bonnand ${ }^{1}$ | \\ D. Pacou ${ }^{1} \quad$ L. Thiercelin ${ }^{1}$
}

${ }^{1}$ DMAS, Onera, F-92322 Châtillon, France

${ }^{2}$ DTIS, Onera, F-91123, Palaiseau, France

Correspondence

Sylvia Feld-Payet PhD, DMAS, Onera,

F-92322 Châtillon, France

Email: sylvia.feld-payet@onera.fr

Funding information

Onera: PRF MECHANICS; CNRS, Grant

number: ANR-14-CE07-0037
The identification of crack growth models requires the knowledge of the crack path geometry and, in particular, of the crack tip position. The objective of the present paper is to provide a criterion for the detection of the crack tip position regardless of the loading conditions or the material behavior. The novelty of the proposed approach is its ability to provide a good estimation of the crack tip even when large plastic deformations occur. The proposed empirical criterion uses the evolution of the displacement gradient standard deviation along a potential crack path to locate the crack tip. It thus relies on full field measures that are relatively smooth while providing accurate displacement vectors even close to the lips. We successfully apply the proposed criterion in two cases of fatigue crack growth: A straight crack at high temperature and a curved crack at room temperature involving large plastic deformations.

\section{KEYWORDS}

crack tip detection, DIC, large deformation

Abbreviations: DIC, digital image correlation; LEF, linear elastic fracture; FE, finite element; DCPD, direct current potential drop method; OF, optical flow; CV, Computer Vision; TV, Total Variation. 


\section{1 | INTRODUCTION}

\section{1 | Problem statement}

Within the framework of aerospace structures' safety, the assessment of the residual lifetime in the presence of a crack is a challenging question. Crack growth predictions require accurate models, which are mainly established in laboratories, on rather simple specimens, by observing the crack tip ${ }^{1}$ evolution. Automatic determination of crack tip location is thus a major issue, but it has only been addressed within a limited framework.

Indeed, crack growth studies mainly consider the stable regime of fatigue crack propagation. This regime very often corresponds to the so-called Paris regime [27], which can be described by Linear Elastic Fracture (LEF) mechanics. In this case, the crack growth rate can be described as a function of the stress intensity factors. Nevertheless, LEF mechanics ceases to be applicable when the failure is preceded by large plastic deformations. Failure is then no longer characterized by the stress intensity factors, but by other parameters, such as the Crack Tip Opening Displacement [39] or the J-integral [28], which correspond to the Non-Linear Fracture Mechanics framework. In any case, all the fracture parameters are related to the crack tip. However, to the authors' knowledge, there is no agreed-upon solution to determine crack tip location when large plastic deformations occur.

The objective of this work is to propose a new methodology for automatic detection of the crack tip based on estimated dense displacement fields. This approach is meant to be versatile, in the sense that it should work:

- Regardless of the loading conditions (i.e., mode I or mixed mode crack growth, monotonic or cyclic loading);

- And regardless of the material's constitutive behavior, in particular of the plasticity level and failure mode (i.e. ductile or brittle).

Notably, the proposed strategy should be robust enough to deal with materials with not perfectly straight crack paths (due to the loading conditions and/or to the microstructure) and blunt crack fronts.

\section{2 | Related Work}

\subsection{1 | Prerequisites: potential crack path detection}

Determining the crack path is a necessary first step before crack tip detection. For engineering structures, most approaches rely on direct processing of the images in order to enhance the contrast of the crack and to clean its shape using morphological operations (e.g. in [34]; see [25] or [40] for a review). However, the contrast is highly dependent on the material, the handling of its surface and the illumination system. Besides, these segmentation techniques are limited in their spatial resolution to the pixel level (i.e. these techniques are not able to capture cracks when the lip opening is less than one pixel). Consequently, portions of the crack could be ignored. More details on the crack can be obtained by comparing images between the current image and a reference image with a smaller crack, notably thanks to Digital Image Correlation (DIC) [30].

Several crack path tracking techniques proposed in the literature rely on a minimization guided by the DIC residual map. Within the X-DIC framework, Réthoré et al [33] use a two-step minimization of the correlation residual by first approximating the crack by a straight line, then optimizing the shape of the crack using a level set approach.

${ }^{1}$ Let us note that the authors use the term "front" for a general designation of the complete line (in three dimensions) and use the term "tip" to designate the visible point corresponding to the intersection of the crack front with the surface. 
A similar approach is used by Fagerhold et al. [9] within the ductile failure framework. Validation of the proposed numerical tools is performed on synthetic data only and is limited to the crack path optimization and not the crack tip determination (the initial guess for the crack path is the exactly known crack path with a sinus variation along its length).

Gregoire [13] uses local DIC and compares local displacement measurements on diagonally opposite points in the elements of a square grid to locate the crack. However, only a first rough approximation of the crack path is obtained, especially for mixed mode propagation, because displacements originating from local DIC algorithms are inaccurate close to the crack. Indeed, in local DIC algorithms, displacements are often imposed to be uniform or to vary linearly within a window. This assumption is not valid when the crack path crosses the considered window. In keeping with [33], Gregoire then adopts an extended finite element approach and refines the crack path by optimization.

In all previously described approaches, the initial model of the displacement field is defined on a grid coarser than the pixel size and thereby is not able to accurately capture the crack path. Once a crack path is determined thanks to this first evaluation, a second step optimization has to be conducted to better capture the discontinuous displacement fields near the propagating crack. For example, to improve the estimation provided by an isoparametric Q4 DIC algorithm near a crack, Fagerhold et al. [9] define overlapping elements in a region containing the crack and use a binary mask, which can be defined at the spatial resolution of a pixel, to separate the nodes belonging to each side of the crack.

More recently, Turner [37], proposed, for brittle materials, a two-step process to replace the DIC measure, in areas with poor correlation or aberrant results, with peridynamics-calculated displacements. This computation requires the knowledge of a linear-elastic material law and the choice of a critical bond stretch to produce a damage field. However, contrarily to the previously mentioned papers, no discrete crack path was computed from these displacement measures.

In contrast, we use optical flow estimation methods recently proposed in the computer vision community [6, 20, $5,38]$ that are able to capture large motions (consequently also suitable within the ductile failure framework) while providing accurate displacement vectors even close to the lips of the crack in one step, without any knowledge of the constitutive material. These methods all rely, to a certain point, on a Total Variation-like regularization, which offers both a smooth displacement field far from the crack and highly resolved strong gradients around the crack. These are particularly important features to exploit data near the crack path and the crack tip, which is an essential part of this study.

Then, from the obtained displacement field, we compute a potential crack path computed by following the maximum gradient of the regularized dense displacement fields with the Marching Ridges algorithm proposed by Feld-Payet et al. [10]: This algorithm provides a curve which is an accurate estimate of the crack path. It should be emphasized that the proposed approach does not assume a priori knowledge of the crack path form or orientation. However, if this is a reasonable assumption, a straight line can also be considered as a potential crack path.

No further optimization step for the displacement field or the potential crack path is performed in this work.

\subsection{2 | Crack tip detection}

Classically, once the crack path is known, the crack length is evaluated by the Direct Current Potential Drop (DCPD) method. This evaluation requires a calibration step and can be biased by the presence of high plastic strain, leading to inaccuracies in the determination of the crack length and thus the stress intensity factors. This method is thus 
restrained to small-scale yielding conditions. It is usually applied to straight cracks. In spite of its limitations, the DCPD method remains up to now the reference for crack length estimation. A recent work [18] offers a comparison between DCPD and DIC-based methodologies.

When using DIC results, the crack tip position is either determined manually $[17,26]$ or using a LEF mechanics analytical solution for the displacement field. Yoneyama et al [41] use an iterative procedure based on the NewtonRaphson method to determine the crack tip location and the mixed-mode stress intensity factors at the same time. According to Réthoré [31], a more robust approach consists in relying on the concept of an elastic crack tip which is detected using the first super-singular term of the William's expansion [14, 15, 22, 32, 8, 31]. This approach requires the delineation of the process zone, which is omitted during the identification of Williams' expansion. It gives an equivalent crack tip position estimated from the far elastic field. In practice, the observed crack tip position can be different from the equivalent position, especially if the process zone around the crack tip is relatively large. This might explain why error studies are mainly performed on quantities such as the stress intensity factors, but rarely concern the crack tip position evaluation. Another reason may be the absence of a reference crack tip position and the difficulty to generate representative synthetic data. To the authors' knowledge, validation on synthetic data for crack tip detection is quite rare (unlike validation on synthetic data for crack path optimization and displacement measurement accuracy). On that issue, it is worth mentioning the analysis of Réthoré in [31] using a DIC technique augmented by an elastic regularization (combining finite element kinematics and a truncated William's expansion). In this case, the displacement field used to deform the reference image consists of a mode I singular field. With these consistent choices of the imposed displacement field and the form of the sought after solution on the one hand, and the exploitation of knowledge of the elastic constitutive behavior on the other hand, the author obtains excellent consistency between the reference crack tip and the estimated one. However, most studies are based on experimental data and thus do not have any exact reference crack tip position. The error analysis then only mention a crack tip position uncertainty. For example, the review by Tong [36] reports one uncertainty analysis on crack tip position by Hamam et al. [14] who mention that their 10-pixel uncertainty is considered as standard. Moreover, the fact that only the far elastic field is analyzed prevents the application of this approach within the generalized plasticity framework.

To find the position of the crack tip independently of the constitutive behavior of the studied material, another option consists in thresholding the displacement gradient magnitude so as to distinguish high displacement gradients associated to the crack lips from regions of lower gradients that correspond to (possibly plastic) strains in the process zone ahead of the crack tip. This is essentially the approach proposed by Grégoire [13] in keeping with his work on crack path localization. Through discretized displacement gradient thresholding, he classifies regions (or patterns) of the material as "broken" (i.e. containing a portion of the crack) or "unbroken". The crack tip is then defined as the center of the broken pattern surrounded by the smallest number of broken patterns. The method highly depends on the classification step: Depending on the chosen threshold, regions affected by a continuous strain can be considered as broken patterns and lead to a mislocalization of crack tip. The threshold is proportional to the mean value of the considered estimate of the displacement gradient over the whole image, and Grégoire mentions various techniques to regularize the process such as morphological operations to eliminate scattered incorrect broken patterns or the use of a mask to reduce the "effective thresholding area". Let us underline that Grégoire specifies that his method is restricted to brittle failure analysis. To validate his methodology, he considers a synthetic image deformed directly by imposing analytical displacement fields from the LEF mechanics framework and obtains the difference vector $[2,2] \pm 4$ pixels. An additional optimization can be performed based on the knowledge of the sought after solution.

In this paper, similarly to Grégoire's work, the authors determine the crack tip position by thresholding some local measure based on the displacement gradient. However, a major difference with [13] is that the threshold value is composed of two terms evaluated respectively along the crack path and right ahead of the crack path. It will be shown 
that this key feature allows the proposed methodology to be versatile and not limited to brittle failure.

\subsection{3 | Validation strategy}

This paper proposes a new crack tip detection criterion also applicable to cases featuring large plastic deformations, whereas other techniques rely on the elastic fields away from the crack tip to provide only an equivalent position. This work relies on the exploitation of the displacement gradient standard deviation near the crack tip. It is therefore important to have relevant data. This is why this work takes advantage of real experimental images: In reality, the gradients' distribution may be different from what could be captured by a numerical model, not only due to the material model, but also due to the discretized representation. The results are then compared with the ones obtained thanks to another experimental technique: The Direct Current Potential Drop (DCPD) method. If the crack opening is too large and the DCPD is no longer suitable, the visually observed crack tip is then used as a reference. Since the DCPD method relies on current conduction, this study considers only metallic specimens and an opening crack mode. Two fatigue tests performed in different conditions and with different crack path geometries are considered to illustrate the proposed methodology's versatility. One of them challenges the proposed criterion in a high temperature context and the second experiment involves a significant plastic flow at the end of the test, which allows to test the criterion when plasticity is not confined near the crack tip. The same set of parameters leads to good results for both cases.

Finally, in order to assess the performance of the proposed methodology in a case where the reference crack tip position is known, a linear-elastic case based on synthetic data (generated from a finite element computation representing the second experiment) is considered. Given the finite element discretization error and the fact that no information about the material is used, a difference of less than 10 pixels validates the methodology.

\section{3 | Paper outline}

The paper is organized as follows. First, in Section 2 we present the experimental procedure and the experimental setup for the two fatigue tests, as well as the synthetic data case, since it is directly based on the second fatigue test. Then, in Section 3 we explain the bases of the crack tip detection criterion. We also perform a parameter study on experimental data to recommend a particular set of parameters and assess the robustness of the choice. In order to only evaluate the influence of the parameters, the same potential crack path is used for all the images of each experiment. Then, with the selected set of parameters, we evaluate the crack tip detection criterion on experimental and synthetic data, for different crack paths, in Section 4. The analysis of the three cases allows us to validate the proposed criterion. Finally, we conclude in Section 5 with a summary of the most important features and some propositions for related future studies. Appendices A and B describe, respectively, the selected optical flow estimation method and the crack path tracking algorithm used for the experimental cases.

\section{2 | EXPERIMENTS}

Two crack propagation tests are performed on a servo-hydraulic fatigue device with $\pm 100 \mathrm{kN}$ of full capacity. Motivation for performing the test is to show the possibilities of the developed approach in two different cases. In the first experiment, a straight crack path is observed in a high temperature context on the Co-base alloy called HA188, which is widely used in combustion chamber applications. The second experiment consists of a test performed on a perforated $316 \mathrm{~L}$ stainless steel specimen at room temperature. The presence of a perforation leads to a curved crack 
path to a significant area with plastic deformations at the end of the test. Both tests are force-controlled at positive load ratio.

\section{1 | Experimental procedure}

The fatigue crack length is determined by the DCPD method in accordance with ASTM Standard E647 [1]. The principle of this technique is based on an electrical current flowing through a conducting specimen and the measurement of the electric potential difference across the crack. The accuracy of this method mainly depends on the current leads and potential probes locations, and consequently on the calibration curve. Classically, the calibration curve comes from another experimental method such as beach marking or from an analytical solution valid for simple geometry, as the Johnson's formula [19]. However, restrictive geometry assumptions are involved. Indeed, the calibration curve associating the electrical potential difference and the crack length is restrained to $2 \mathrm{D}$ configurations, since it considers only a straight line perpendicular to the current lines, and a straight front across the thickness. To overcome these limitations for complex geometries, it is possible to resort to numerical calibration by the Finite Element (FE) method. Initiated by Ritchie and Bathe [29] and followed by Hicks and Pickard [24], this approach only requires the knowledge of the crack path. Taking into account the front curvature is then possible as described in [11].

In practice, the DCPD acquisition is triggered at the maximum loading point in order to avoid crack closure effect. A first estimation of the crack length is obtained thanks to Johnson's equation. If necessary, this measurement can be refined by performing an a posteriori FE simulation taking into account the final observed crack path.

Finally, a camera with a definition of $1936 \times 1216$ pixels completes the setup. The lens allows reaching a pixel size of 20 microns. The camera is also synchronized with the maximum of force. To limit the number of images, special attention is paid to the acquisition sequence. Image recording is triggered by the overrun of some predefined threshold on the crack growth (the length being provided by the DCPD method combined with an a priori calibration).

\subsection{Experiment 1 at high temperature}

Experiment 1 was carried out on a plate specimen made of the Co-base alloy at $600^{\circ} \mathrm{C}$. The test configuration is a Single-Edge-Notch specimen with a $3 \times 45 \mathrm{~mm}$ cross-section. The notch is $0.3 \mathrm{~mm}$ long and $0.1 \mathrm{~mm}$ wide. The specimen was covered with a speckle pattern obtained by layering white dots with a boron nitride spray over a commercial black paint resisting up to $700^{\circ} \mathrm{C}$. The specimen was heated by a middle-frequency induction system, as shown in Figure 1. Preliminary tests (with and without air flow) have shown that heat haze does not impact displacement measurement in this case.

In order to assess the frequency effects on crack growth, not discussed in this paper, a sequential testing procedure was adopted. The loading changes were triggered automatically based on the estimation provided by the DCPD method combined with a priori calibration of the crack length. As recommended in [1], the procedure started with a fatigue precracking step during which the crack length grew from $0.3 \mathrm{~mm}$ to $3.5 \mathrm{~mm}$. Then, four steps were performed under a nominal stress of $300 \mathrm{MPa}$, as described in Figure 2. First, successive modifications of the triangular cycle frequency were applied, from $1 \mathrm{~Hz}$ up to $8 \mathrm{~mm}$ crack length, then $0.25 \mathrm{~Hz}$ up to $11 \mathrm{~mm}$ and $0.025 \mathrm{~Hz}$ up to $15 \mathrm{~mm}$. Finally a cycling with a hold-time $10 \mathrm{~s}-10 \mathrm{~s}-10 \mathrm{~s}$ was performed. 


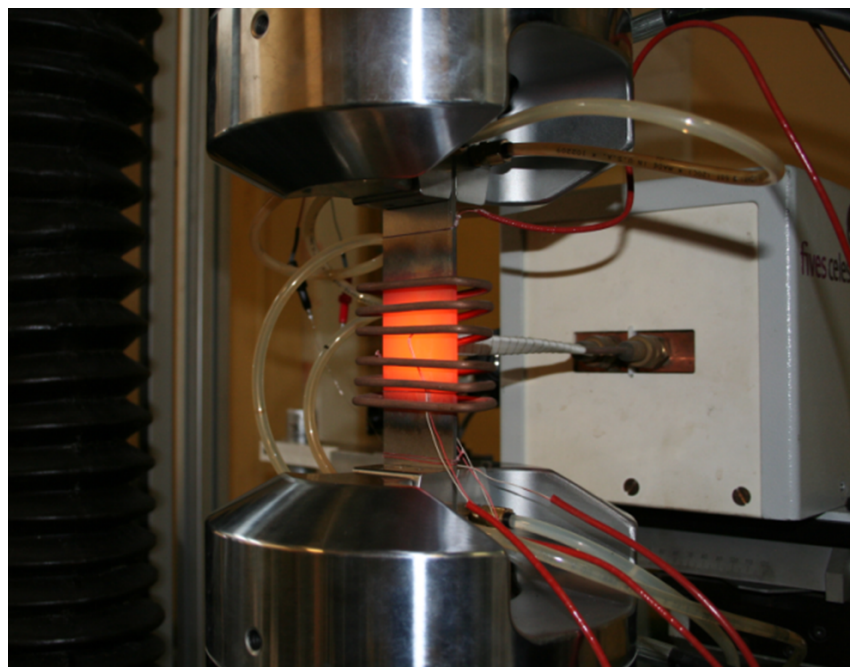

FIGURE 1 Setup of Experiment 1, with HA188 specimen heated by induction unit.

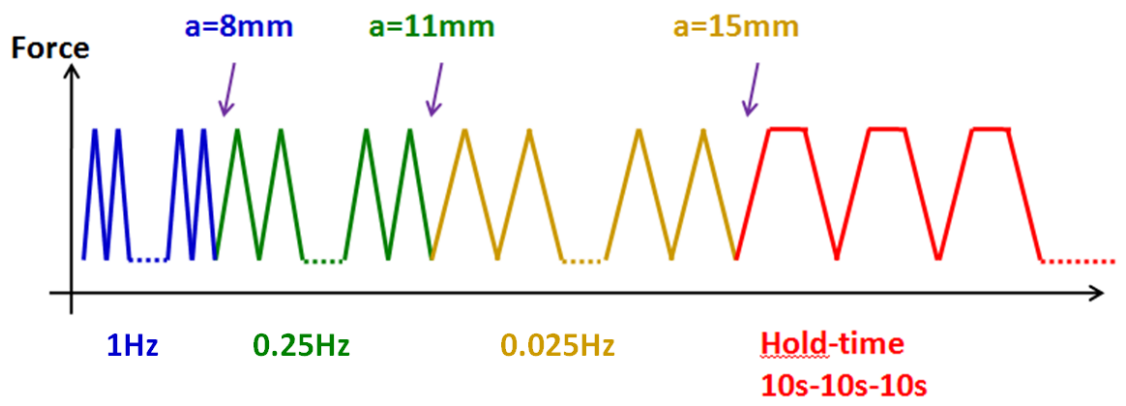

FIGURE 2 Experiment 1: Loading sequences based on electrical measurement applied on HA188 specimen.

\section{3 | Experiment 2 on a perforated specimen}

The considered specimen is made of $316 \mathrm{~L}$. This austenitic stainless steel is widely employed in aeronautics, pressure vessels and nuclear power plant, namely for its high temperature resistance corrosion and its ductility. A schematic description of the geometry is given on Figure 3.

Prior to the fatigue test, the specimen was sprayed with a thin coat of matte black paint and afterwards with a dispersed commercial white paint. During the test, no external parameter was modified, i.e. no pre-cracking step was performed. Constant load amplitude corresponding to a nominal stress of $135 \mathrm{MPa}$ was applied at a frequency of $2.7 \mathrm{~Hz}$. The loading ratio was $R=0.1$. The fatigue test was stopped after 121852 cycles; based on the deformed geometry, the final crack length was then estimated at $37.75 \mathrm{~mm}$ (from the notch side).

The experimental crack path broadly corresponds to a classical pure mode I crack but, as expected, the presence of localized stress gradients near the perforation caused a slight deviation (see Figure 4, on the left). Surprisingly, the comparison of the calibration curves obtained by an electrical simulation taking into account the real crack path and 


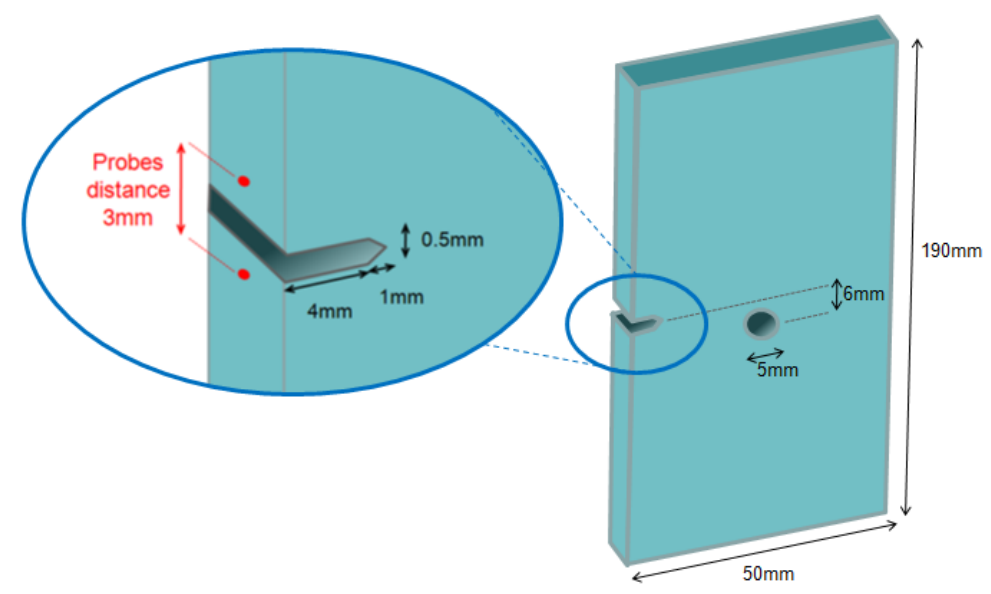

FIGURE 3 Geometry of the perforated specimen used in Experiment 2.

an analytical formula based on a straight crack indicates that this kinking has virtually no effect on the estimation of the crack length and only a moderate impact on the stress intensity factor (see Figure 4).
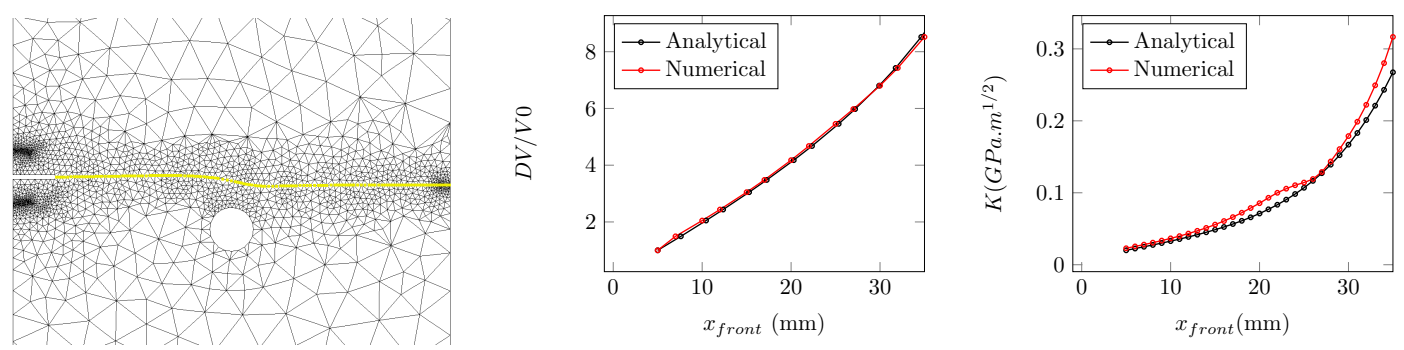

FIGURE 4 Experiment 2: On the left, a portion of the mesh with the real crack path in yellow. In the middle, the DCPD calibration curves obtained by using analytical calibration formula based on a straight crack (in black) or numerical calibration taking into account the real crack path (in red) are very similar. Taking into account the real crack path geometry has only a moderate impact on the corresponding stress intensity factor, on the right.

\subsection{Limits of the reference measure}

The horizontal position determined with the DCPD method serves as a reference to validate the results obtained with the proposed criterion. However, this method is associated with a certain number of requirements which may not always be met. For example, this method requires a straight crack front to be accurate, but this may not be the case when the crack is short and just starting to expand (i.e. during the precracking step), as evidenced in the Experiment 1 by the fractography shown in Figure 5. Considering small cracks, Doremus et al. [7] have shown that the front curvature has an impact on the DCPD results: It causes a bias in the electrical measurement by a $2 \mathrm{D}$ analysis. Here the required correction in the calibration was not performed since precracking was not considered in the related crack growth rate analysis. In the absence of any reference results for short cracks, all results are to be compared from the 
moment where crack propagation is stable and the crack front is supposed to be straight.

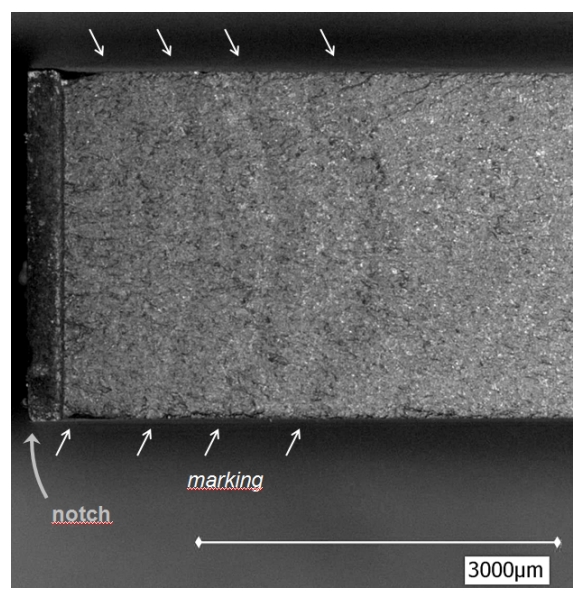

FIGURE 5 View with an optical microscope of a crack surface for Experiment 1 on HA188 material: The decrease of mechanical loading per stage modifies locally the roughness of cracked zone, inducing a mechanical marking. The visible crack fronts show a significant front curvature at the beginning of the test.

Besides, the 2D analysis for the DCPD method does not take into account the fact that the crack opening may be relatively large (see Figure 6). Consequently, during the last stage of failure for the perforated specimen, the DCPD method cannot serve as a reference. However, as the crack tip is rather blunt, its position can at least be visually estimated.

\subsection{Synthetic data case based on Experiment 2}

The DCPD method being error-prone, synthetic data are used to provide a trustworthy reference crack tip position and finally validate our proposition.

The synthetic image generation is obtained thanks to a numerical tool very recently proposed by Bouda et al. [2]. However, in this paper, instead of a grid, the images' texture is the speckle pattern from the reference image of Experiment 2. The first image corresponds to the initial specimen geometry with only a pre-notch. The second image is obtained by deforming the first one with a displacement field, which contrarily to studies in [13, 31], is not derived from an analytical formula. The displacement field results from a linear elastic finite element computation with a propagated crack (of a length close to what is observed in image 8 for Experiment 2), see Figure 7. The input data are those used for the numerical calibration of the DCPD method for Experiment 2.

Let us note that the crack does not exactly propagate along a straight horizontal line. For the considered loading, the horizontal progression of the crack from the tip of the pre-notch is about $15.00 \mathrm{~mm}$ while its vertical progression is about $0.06 \mathrm{~mm}$. The pixel resolution being approximatively $0.02 \mathrm{~mm}$, this change already has a slight impact on the deformed image. 

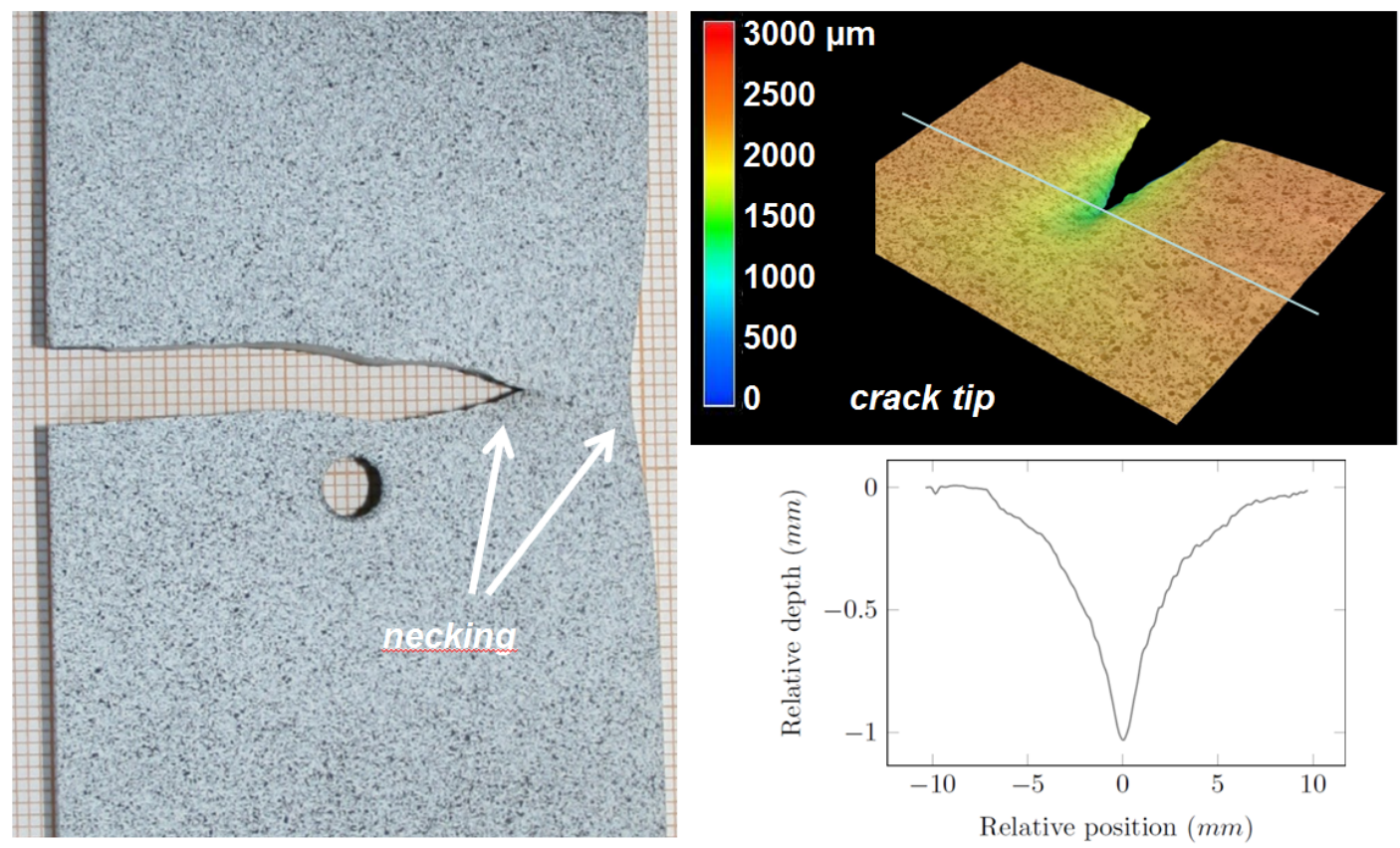

FIGURE 6 In Experiment 2, necking occurs at the end of the test: In the horizontal direction, as visible at the macroscopic scale (on the left) and in the out of plane direction, as noticeable thanks to a digital microscope (on the right): Up to $1 \mathrm{~mm}$ thickness decrease can be measured, compared to the original $4 \mathrm{~mm}$ thickness.

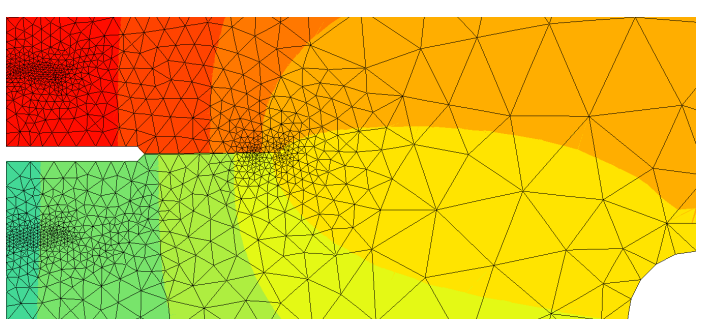

0

$u_{y}(\mathrm{~mm})$

0.107

(a) Vertical displacement field in the cracked area.

FIGURE 7 The synthetic images take advantage of the speckle pattern from Experiment 2 and a FE analysis on a cracked geometry.

(b) Deformed synthetic image

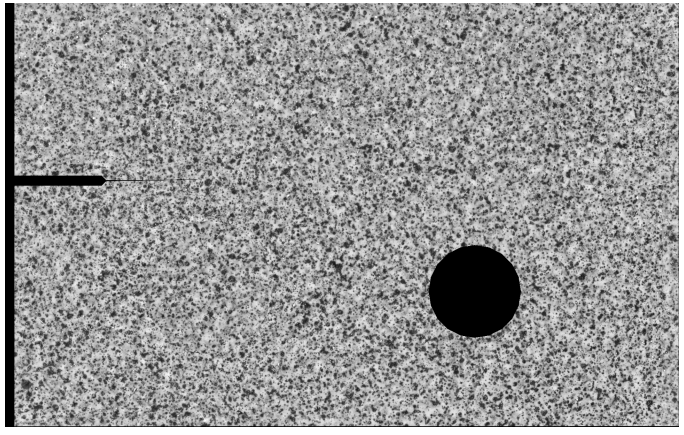




\section{3 | CRACK TIP DETECTION}

This section aims at detailing the crack tip estimation process, which involves the choice of some parameters. First, we determine a set of parameters which could serve as a basis for a following analysis and evaluate the robustness of this choice. We select these parameters in order to obtain a smooth crack length evolution that reduces the discrepancy with the DCPD measure in the loading range where it is supposed to provide valid results. Once a set of parameters is determined for all experiments, the detailed analysis of the remaining local differences is given in Section 4.

\section{1 | Prerequisites}

First, the data necessary to the use of the proposed crack tip detection criterion are specified.

\subsection{1 | Dense displacement field estimation}

The crack tip detection criterion relies on a dense displacement field that must be able to:

1. Allow an accurate localization of the crack lips;

2. Provide a relatively smooth displacement field further away from the crack;

3. Capture large displacements.

Several works in Computer Vision (CV) have studied these issues since the 90's. Point 1. and 2. are usually addressed by defining the estimated field as the minimum of a compound criterion (in keeping with the seminal work of Horn and Shunck [16]) and by adopting a Total Variation-like regularization [5, 20]. TV regularization is well-known in various inverse problems to efficiently provide piecewise smooth solutions. As for estimating large displacement (point 3.), most methods use a coarse-to-fine estimation based on a Gaussian pyramid of images derived from the original ones [5, 20,38]. These approaches are now classical in the CV domain and are provided in various software packages, such as OpenCV, an open library publicly available and usable within a python framework for instance. Let us emphasize that these are "phenomenological solutions" which are not related to a physically sound model of the displacement field: Indeed these methods are used in various contexts, very different from full-field measurement, such as segmentation of moving objects for autonomous urban navigation. However, the authors have found them quite useful to provide a displacement field compatible with points 1.-3. Hence, hereafter we use the DeepFlow algorithm [38] available in OpenCV. More details on this method, and a comparison with standard window-based techniques, are provided in Appendix A.

\subsection{2 | Potential crack path}

It is assumed that the search for a crack tip is restricted to a potential crack path, similarly to the approach of Réthoré in [31]. This potential crack path can be known in advance, e.g. if the crack path is supposed to propagate along a straight line in the same direction as a notch (see Figure 8), or it can be determined thanks to a crack path tracking algorithm for more complex cases.

In this work, both options are considered. The main requirement is that the potential crack path should provide a relatively good approximation of the crack on the reference image: The proposed crack tip detection criterion should be robust enough to withstand a small error. A straight crack path is used for the synthetic data analysis since it is 


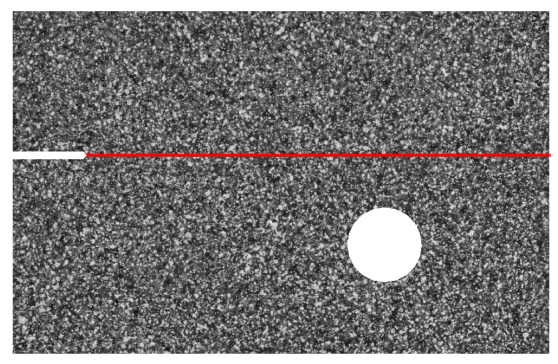

FIGURE 8 Straight potential crack path assumed from the notch's geometry for the reference image of the synthetic data case based on Experiment 2.

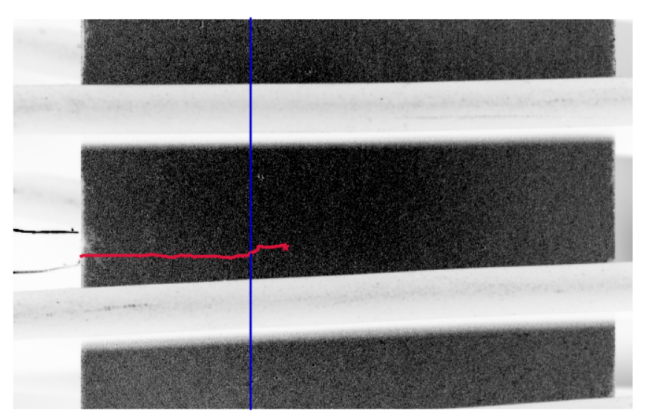

(a) Experiment 1: Image 214

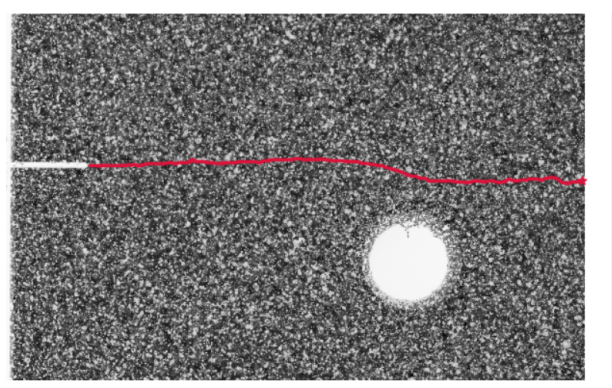

(b) Experiment 2: Image 214

FIGURE 9 Final potential crack path with the penultimate measure of the crack length with the DCPD method traced on the first image.

a reasonable approximation in this case. As for the experimental data, the potential crack path is obtained thanks to the Marching Ridges algorithm proposed by Feld-Payet et al. (see [10] for more details on the original algorithm). Its principle is to approximate the ridge line of a scalar field that presents a local extremum (maximum or minimum) at the crack lips: Here the first derivative of the displacement norm is considered. The crack path is build incrementally image by image, leading to what is referred to as the final crack path for the last image. For more details, see Appendix B.

In order to distinguish the effects of the potential crack path tracking strategy and those of the proposed criterion, all the results in Section 3 on the crack tip detection consider only the final potential crack path, i.e. the one obtained for the last process image: See Figure 9. However, in Section 4, both crack paths (current and final) are considered in order to better assess the complete methodology: The crack path determined on the currently studied image may punctually be more challenging than the final one.

\subsection{3 | Combination of the initial data}

The potential crack path serves as a basis for the crack tip detection. Several options may be considered for the crack path tracking as long as the end results lies relatively close to the pixels corresponding to high displacement gradients. To evaluate the relevance of the estimated crack path, its position may be compared to the map of the maximum gradients of the displacement norm, $g_{m}$, which is simply approximated by finite difference from the displacement 

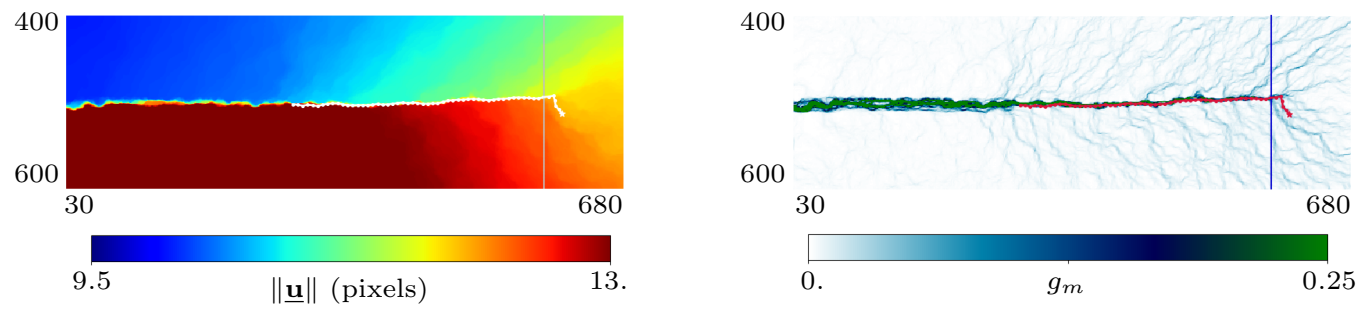

FIGURE 10 Left: Displacement norm; right: Maximum gradient map. Potential crack path obtained with the Marching Ridges algorithm for image 8 of Experiment 2. The crack path first follows a horizontal line of large gradient values, which corresponds to the crack opening but ends up beyond the DCPD estimated crack tip position marked with a blue vertical line to follow a branch, which corresponds to smaller gradient values.

norm on adjacent pixels:

$$
g_{m}=\max \left(\left\|\underline{\mathbf{u}}_{i, j+1}-\underline{\mathbf{u}}_{i, j}\right\|,|| \underline{\mathbf{u}}_{i+1, j}-\underline{\mathbf{u}}_{i, j}\|,\| \underline{\mathbf{u}}_{i+1, j+1}-\underline{\mathbf{u}}_{i, j}\|,\| \underline{\mathbf{u}}_{i-1, j+1}-\underline{\mathbf{u}}_{i, j} \|\right)
$$

where index $i$ designates the line number and index $j$ designates the column number on an image, see Figure 10. The correspondence before the crack tip is the basis of the evaluation of the crack tip detection criterion.

\section{2 | Bases of the proposed criterion}

\subsection{1 | Theory}

To determine the crack tip position along a potential crack path, a new criterion is proposed. This criterion is based on the hypothesis that the crack should correspond to relatively high and localized gradients (which should be captured by an appropriate DIC algorithm) whereas the crack tip should mark a transition with a smoother distribution of relatively lower displacement gradients. To study this shift in the gradient value and distribution, the authors propose to consider the evolution along the potential crack path of the maximum gradient between adjacent pixels, $g_{m}$ (as defined in equation 1), in terms of local standard deviation: $\sigma_{g m}$.

\subsection{2 | In practice}

\section{Normalization}

Before formulating this criterion in algorithmic terms, it is important to note that the gradient values should increase as the crack propagates. Indeed, according to LEF Mechanics, the stress intensity factors (and thus the elastic part of the displacement gradients) increase with the crack length. Consequently, to formulate the criterion, the authors propose to consider the evolution of the gradient maximum standard deviation divided by its mean value along the potential crack path. The result of this division by the mean value will be referred to as normalized and will be denoted by $\bar{\sigma}_{g m}$ in this paper.

\section{Local gradient maximum standard deviation}

To determine a local gradient maximum standard deviation, the authors propose to consider, around an evaluation point, a relatively small window. To evaluate the influence of the window size in the crack tip determination, the 

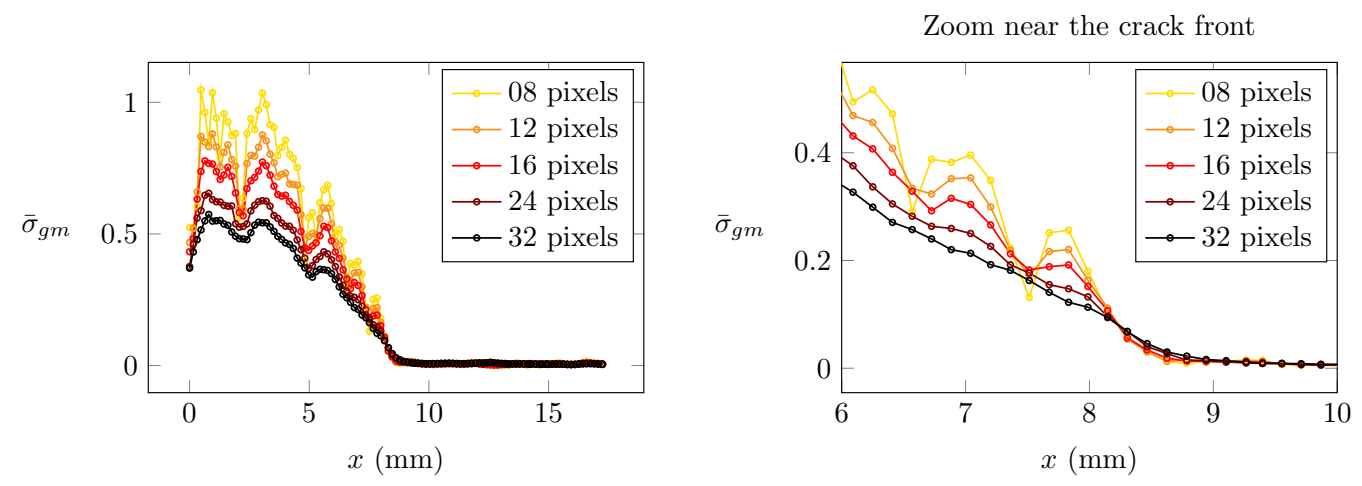

FIGURE 11 Evolution of the normalized standard deviation $\bar{\sigma}_{g m}$ along the potential crack path for Experiment 1 at high temperature, for image 115 , along the whole potential crack path (left) and especially near the crack tip (right). The specified values correspond to the size of the considered windows. Windows between 12 and 16 pixels seem to provide smooth enough curves which present a sufficiently sharp drop.

evolution of the normalized standard deviation $\bar{\sigma}_{g m}$ along the final potential crack path (see Figure 9 a) is presented for the Experiment 1 at high temperature on Figure 11 for different window sizes. The choice of the window size appears to be a compromise. On the one hand, the window should be small enough so that the drop in the $\bar{\sigma}_{g m}$ values, which corresponds to the crack tip position, is sufficiently sharp. On the other hand, the window size should be large enough so that the evolution is sufficiently smooth to be representative of a tendency. These requirements seem to be met by window sizes between 12 and 16 pixels. A window size of 16 pixels is used in this paper unless specified otherwise. Let us note that the size of the window can compensate for a relative error in the determination of the crack path and thus contributes to the robustness of the method.

\section{Possible approaches}

The authors propose in this paper to determine the crack tip location based on the drop of the $\bar{\sigma}_{g m}$ curve from globally decreasing values to a plateau of relatively small values. An important point here is to note that, in the general case, the smallest values are not necessarily null nor close to zero. Indeed, there might be some gradients ahead of the crack tip which may be associated to more or less confined plastic deformations or, more generally, to material heterogeneities.

Bearing this point in mind, several methods can be used to determine the drop of the $\bar{\sigma}_{g m}$ curve. Two of them are studied in this paper:

1. The crack tip can be defined as the last point corresponding to a non negligible gradient of $\bar{\sigma}_{g m}$ along the potential crack path. In this respect, it can be determined by finding the intersection of the gradient of the $\bar{\sigma}_{g m}$ curve with a minimum value close to zero since $\bar{\sigma}_{g m}$ should stay at a rather constant value beyond the crack tip.

2. The crack tip can be determined by the intersection of the $\bar{\sigma}_{g m}$ curve with a minimum value (possibly non zero) representative of the gradient state ahead of the crack tip.

These two approaches will be used sequentially in the following, the first one providing an initial guess that is refined by the second one. 


\subsection{Threshold 1 on the gradient of $\bar{\sigma}_{g m}$}

\subsection{1 | Principle}

This subsection focuses on the first approach: The crack tip position is determined using a threshold on the gradient of $\bar{\sigma}_{g m}$, referred to as Threshold 1 . This gradient is evaluated by finite difference for each point $\underline{\mathbf{X}}_{i}$ of the potential crack path:

$$
\bar{\sigma}_{g m}, x\left(\underline{\mathbf{x}}_{i}\right)=\frac{\bar{\sigma}_{g m}\left(\underline{\mathbf{x}}_{i+1}\right)-\bar{\sigma}_{g m}\left(\underline{\mathbf{x}}_{i}\right)}{\left\|\underline{\mathbf{x}}_{i+1}-\underline{\mathbf{x}}_{i}\right\|}
$$

The idea is then to determine the last not negligible negative gradient of $\bar{\sigma}_{g m}$ along the potential crack path, before the plateau. The threshold value should be slightly greater than the maximum negligible value. So, ideally, the threshold value should be related to the considered image. That is why the authors propose that the threshold value should be a fraction of the standard deviation along the potential crack path of the absolute value of the considered gradients $\bar{\sigma}_{g m}, x$, denoted $\sigma_{G}$. The crack tip is then defined as the last discrete point (of index $i_{\text {front }}$ ) of the potential crack path where the absolute value of $\bar{\sigma}_{g m}, x$ decreases below the threshold value:

$$
\left|\bar{\sigma}_{g m}, x\left(\underline{\mathbf{x}}_{\text {front }}\right)\right|<\alpha \times \sigma_{G}
$$

This proposition is illustrated on Figure 12 for image 115 of Experiment 1 at high temperature. The latest point corresponding to $30 \%$ of the standard deviation $\sigma_{G}$ (see the horizontal threshold on the gradient curve depicted in the top part of the Figure) has indeed almost exactly the same horizontal position as the one indicated by the DCPD method, as shown by the two vertical bars associated with the $\bar{\sigma}_{g m}$ curve on the bottom.

\subsection{2 | Parameter study}

The impact of the choice of the coefficient $\alpha$ seems to be case dependent. Indeed, as can be seen on Figure 13, a coefficient of $\alpha=0.4$ seems to provide less satisfactory results in terms of estimated crack length for Experiment 2 when the crack passes by the hole (i.e. between images 90 and 140). For Experiment 1, satisfactory results are obtained even with values up to $\alpha=0.7$. However, in both cases, a too low value can also occasionally cause some trouble: e.g. $\alpha=0.2$. A coefficient of $\alpha=0.3$ seems to provide satisfactory results in both cases. This value will be chosen as a default value in the following.

\subsection{3 | Discussion}

With an appropriate choice of the threshold value, this criterion on the gradient of $\bar{\sigma}_{g m}$ allows to have a good approximation of the crack tip position. However, the crack tip position is then quite dependent on the potential crack path discretization since the gradients calculation (equation 2 ) is approximated by finite difference. To alleviate this issue and obtain a more refined estimation, the second approach of thresholding $\bar{\sigma}_{g m}$ can be used as a complementary step, as described in the next section. 

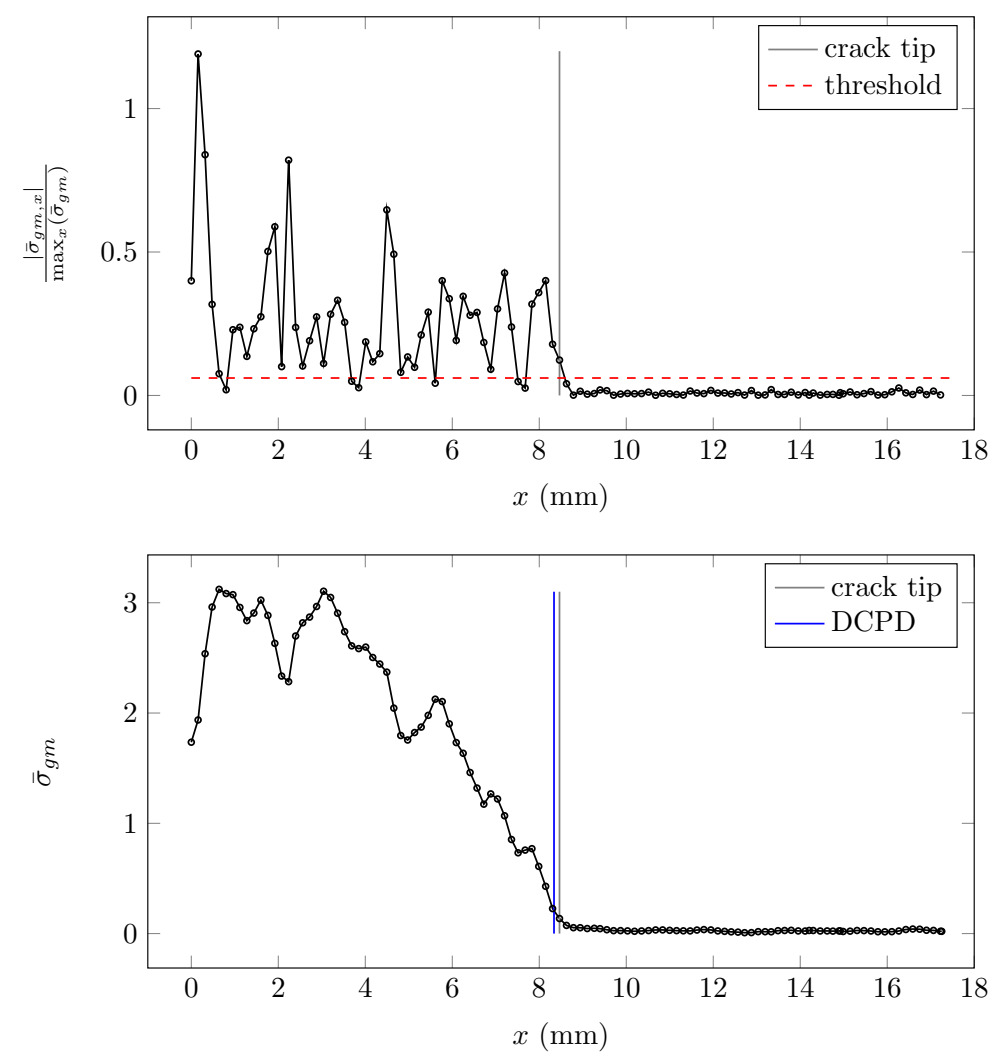

FIGURE 12 Experiment 1 at high temperature, image 115. The crack tip position (in gray) given by the intersection between $\left|\bar{\sigma}_{g m}, x\right|$ and Threshold 1 (in red) corresponding to $30 \%$ of its standard deviation corresponds very well with the one obtained with the DCPD method (in blue).
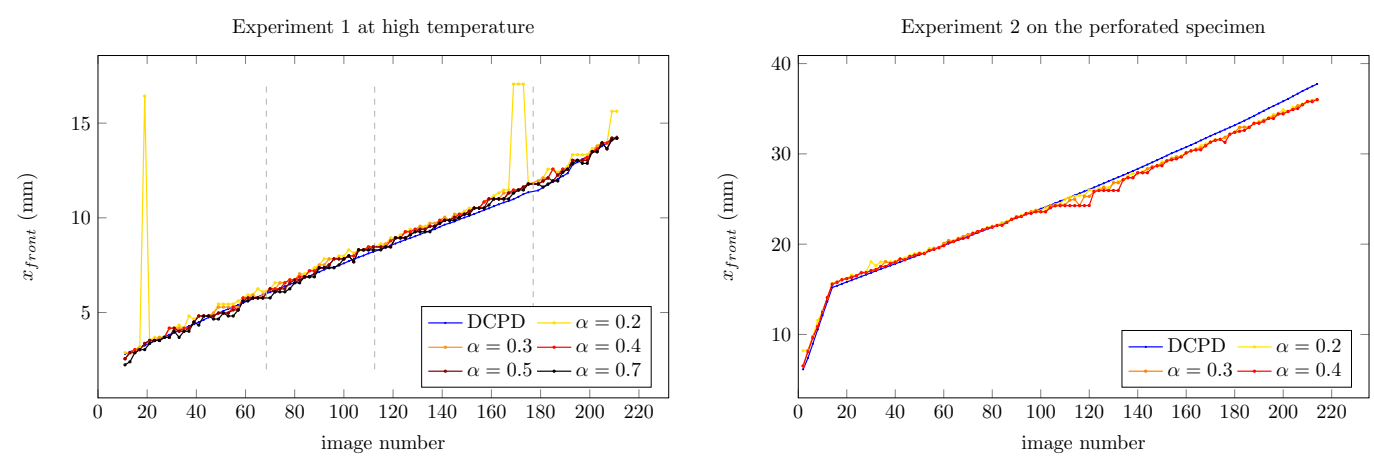

FIGURE 13 Evolution of the crack tip horizontal position with the image number obtained with Threshold 1 for various $\alpha$ coefficients compared with the DCPD measure. Considering $30 \%$ of the gradient of $\bar{\sigma}_{g m}$ as a threshold provides satisfactory results for both cases. 


\subsection{Threshold 2 on the $\bar{\sigma}_{g m}$ curve}

\subsection{1 | Principle}

The idea is to determine, based on the knowledge of a first approximation of the crack tip position (in this case determined with Threshold 1), a threshold value for the $\bar{\sigma}_{g m}$ curve. The threshold should correspond to a value that is significant compared to the values in the continuum medium ahead of the crack tip. To evaluate the maximum gradient standard deviation ahead of the crack tip, evaluation points are distributed along half a circle centered on the crack tip position computed in Section 3.3 as depicted in Figure 14. An interpolated crack tip position $\underline{\mathbf{x}}_{\text {front }}$ is

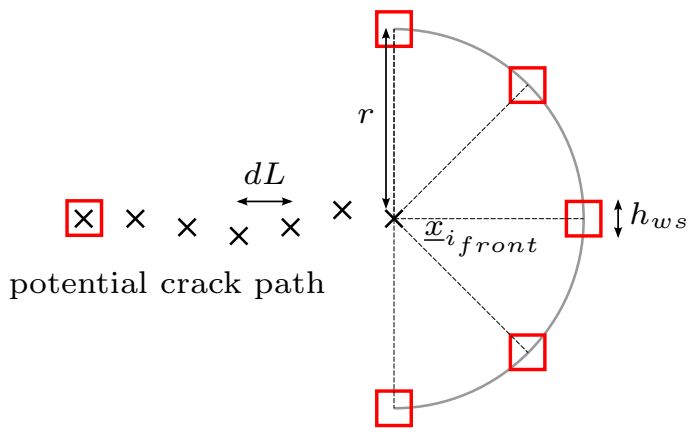

FIGURE 14 Evaluation windows for Threshold 2 on the $\bar{\sigma}_{g m}$ curve: The windows centered at the points of the potential crack path are used to evaluate $\bar{\sigma}_{g m}\left(\underline{\mathbf{x}}_{\text {front }}\right)$; the windows ahead of the crack tip $\left(\underline{\mathbf{x}}_{i_{\text {front }}}\right)$ found with Threshold 1 are used to evaluate the new threshold value. All window sizes are taken equal.

then defined as the intersection point between the $\bar{\sigma}_{g m}$ curve and a threshold corresponding to the maximum value $\sigma_{g m}$ measured ahead of the first approximated crack tip:

$$
\bar{\sigma}_{g m}\left(\underline{\mathbf{x}}_{\text {front }}\right)=\max _{\underline{\mathbf{x}}_{\text {ahead }}}\left(\bar{\sigma}_{g m}(\underline{\mathbf{x}})\right)
$$

\subsection{2 | Parameter study}

Figure 15 presents various crack tip horizontal position estimates obtained with Threshold 2 when varying the radius of the circle $r$ of positions ahead of the crack tip from 2 to 32 pixels for both experiments. The estimation appears not very sensitive to this parameter, which is consistent with the fact that a plateau of similar values is expected near the crack path. A choice of radius of 6 pixels, equivalent to the crack length increment for the Marching Ridges process, is considered in this paper.

The last parameter involved with Threshold 2 is the window size used to evaluate the maximum of $\bar{\sigma}_{g m}$ ahead of the crack tip. It can be taken equal to the one used to evaluate the $\bar{\sigma}_{g m}$ along the potential crack path in order to reduce the number of parameters. Figure 16 presents various crack tip horizontal position estimates obtained when varying the window size $h_{w s}$ from 8 to 32 pixels for both experiments. Larger windows yield smaller values of the maximum standard deviation and consequently lead to higher values of the threshold and to longer estimated crack paths. Window sizes between 12 to 16 pixels provide satisfactory results in terms of crack tip horizontal position.

With windows sizes of 16 pixels with a radius $r$ of 6 pixels, 5 evaluation points allow to cover the half space ahead of the previously determined crack tip with overlapping evaluation areas. 

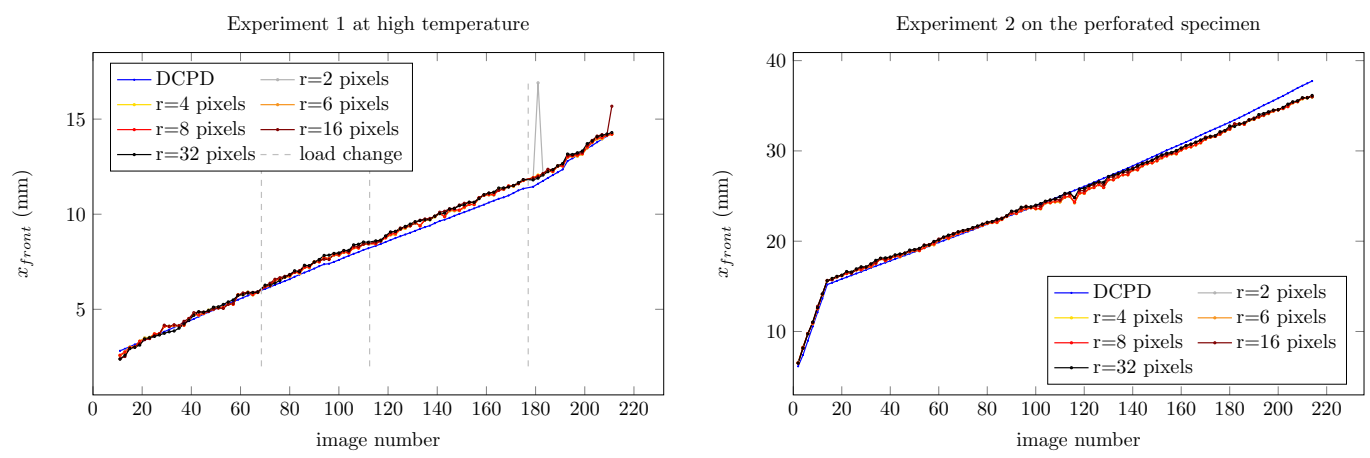

FIGURE 15 Evolution of the crack tip horizontal position with the image number for various radii of evaluation $r$ compared with the DCPD measure. Below 32 pixels, the distance seems to have very little influence on the results.
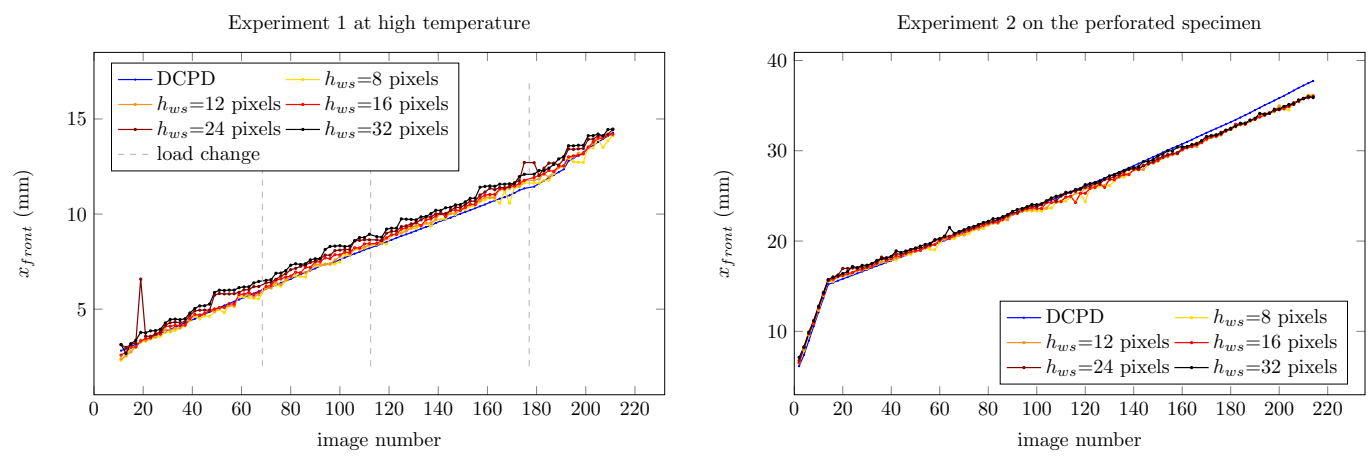

FIGURE 16 Evolution of the crack tip horizontal position with the image number for various window sizes $h_{w s}$ compared with the DCPD measure. The larger the window size, the longer the crack length. Window sizes of 12 to 16 pixels seem to provide better results for the Experiment 1 at high temperature. The impact seems more limited for the Experiment 2 on the perforated specimen.

\subsection{3 | Discussion}

With the chosen parameters, for Experiment 2, the two thresholds are almost equivalent. Indeed, the horizontal crack tip position provided by Threshold 2 is, in average, less than $5 \mu m$ closer to the DCPD measure. This difference only represents $2 \%$ of the distance between two evaluation points (of $223.2 \mu \mathrm{m}$ ), which is negligible.

For Experiment 1, the difference is slightly more perceptible. Although Threshold 2 provides a horizontal crack tip position only $20 \mu \mathrm{m}$ closer to the DCPD measure (which is more than Experiment 2 , but still only about $1 / 8^{\text {th }}$ of the $161.4 \mu \mathrm{m}$ distance between two evaluation points) in average, it allows to diminish the maximum difference by about $277 \mu \mathrm{m}$. This type of correction is necessary only occasionally: Near images $49-51,94-96$, and 185 , as visible on Figure 17.

To conclude, for a small enough distance between two evaluation points (i.e. the center of the windows along the crack path), the choice between the two thresholds is a compromise between computational costs and robustness. Indeed, Threshold 1 involves only 2 parameters (i.e. coefficient $\alpha$ and the window size) and already provides satisfactory results, except very punctually. This punctual errors may be correct thanks to the second step with Threshold 

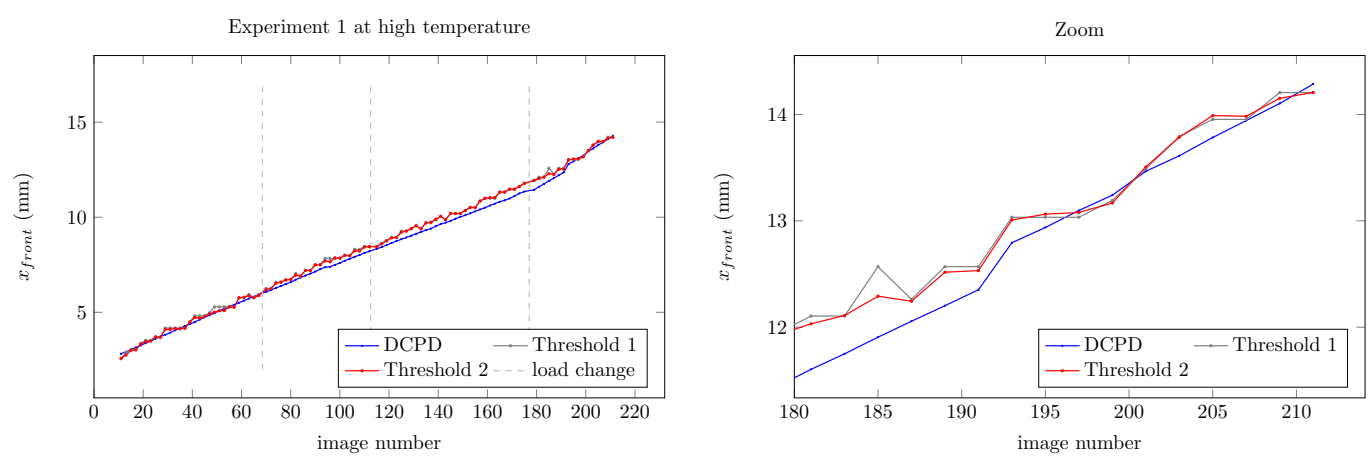

FIGURE 17 Evolution of the horizontal crack tip position (in $\mathrm{mm}$ ) with the image number for Threshold 1 (in gray) and Threshold 2 (in red) compared to the DCPD measure for Experiment 1 . The two thresholds lead to globally equivalent results, except occasionally where Threshold 2 provides a position closer to the DCPD measure.

2, which involves the choice of one more parameter (i.e. the radius $r$ of the half circle), if all window sizes are taken equal. However, this parameter $r$ is found to actually have very little influence on the crack tip position, especially compared to the other two parameters. The number of evaluation points along the half circle can be computed from the radius and the window size so that the windows cover the half space ahead of the crack tip. For both experiments, the beneficial effect of adding a second step with Threshold 2 might be clearer with a larger distance between two evaluation points, which may then also be considered as a parameter. This makes a total of 3 relevant parameters (i.e. $\alpha$, the window size and the distance between two evaluation points). For a different study, the authors recommend to start with the selected set of parameters that already gives satisfactory results for the two different experimental cases (and notably different materials) considered in this paper. In the following, Threshold 2 is considered because of its robustness.

\section{4 | ANALYSES FOR DIFFERENT POTENTIAL CRACK PATHS}

The aim of this section is to evaluate the crack tip detection criterion for the recommended set of parameters, on both experimental and synthetic data, for different crack paths. On a given potential crack path, an approximation of the crack tip is obtained by thresholding the absolute gradient of $\bar{\sigma}_{g m}$, the maximum standard deviation of the normalized local gradient, according to equation 3 , with $\alpha=0.3$. This position is then refined as described in Section 3.4 with 5 evaluation points distributed along half a circle of a 6-pixel radius and a window size of 16 pixels.

\subsection{Summary for the experimental data}

For the experimental data, both the current and final crack paths obtained with the Marching Ridges algorithm are considered. The difference between these two options is that the former corresponds to the data available in the currently studied image, whereas the latter is estimated on the last image in the sequence. The results are presented for the two Experiments in Figure 18. As expected, the results are not identical and, especially for Experiment 1, the crack tip detection for each image shows some local variations which are smoothed when processing the final potential crack path. Indeed if the path obtained with the Marching Ridges algorithm corresponds to the actual crack path followed by the branch of higher gradients, the branch after the actual crack tip may very well differ from one 

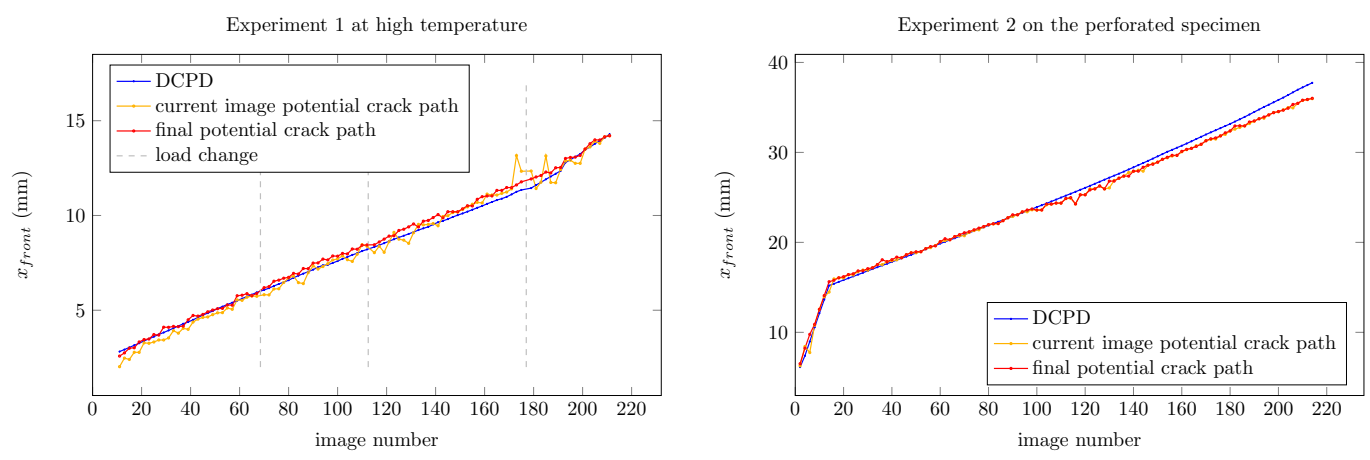

FIGURE 18 Evolution of the horizontal crack tip position (in $\mathrm{mm}$ ) with the image number for the final potential crack path (in red) and a potential crack path estimated for each image (in orange). For both experiments, the two results are globally consistent with the DCPD reference.

image to another. Occasionally, one of these branches of high gradients may lead to a secondary drop of $\bar{\sigma}_{g m}$ but Threshold 1 may not be high enough to discard it. These secondary drops are not observed if the final crack path is considered. The crack paths determined on the current image by the Marching Ridges algorithm are thus particularly challenging for the proposed criterion. Still, both strategies are globally similar and consistent with the reference DCPD estimate for both experiments on different materials, which proves the robustness of the proposed criterion: Detailed comments for each experiment are given below. Based on the the extreme crack tip positions that could be considered as acceptable on the gradient map, the authors estimates that the uncertainty associated to the crack tip estimation is about 10 pixels, which is standard for this type of analysis.

\subsection{Analysis for Experiment 1 at high temperature}

For Experiment 1 at high temperature, the pre-notch is only $0.3 \mathrm{~mm}$ long and several images are available before the crack is long enough for its propagation to be stable. In this case, crack propagation can be estimated as stable for a horizontal position of the crack tip (obtained thanks to the DCPD method) between $4.75 \mathrm{~mm}$ and $5 \mathrm{~mm}$, i.e. for images 45 to 49 . Consequently, the results are to be compared with the DCPD method only from image 49. Propagation seems to remain stable at least up to image 211. After this point, the crack tip position is too close to an induction coil (see Figure 9a), which causes DIC to fail. For image 211, the horizontal position of the crack tip estimated by the DCPD method is about $14,29 \mathrm{~mm}$ and, for the final image 227, about $16.39 \mathrm{~mm}$. A mask has been placed on the induction coil in the maximum gradient map to avoid following the corresponding gradients.

Figure 18 shows that the method applied to the final potential crack path leads to an evolution of the horizontal position of the crack tip in very good agreement with the DCPD measure, despite the load frequency changes. Figure 19 summarizes the estimation at image 115: The estimated (final) crack path appears consistent with both the crack visible in the inspection image ${ }^{2}$ and the gradient map.

The same strategy applied on the potential crack path determined for each image provides a crack length evolution that is globally consistent with the DCPD reference one, as shown in Figure 18. The occasional differences are due to a

\footnotetext{
${ }^{2}$ The crack path projection onto the current image may be less accurate in the area where the crack is more widely open since it relies on the measured displacements between the lips.
} 


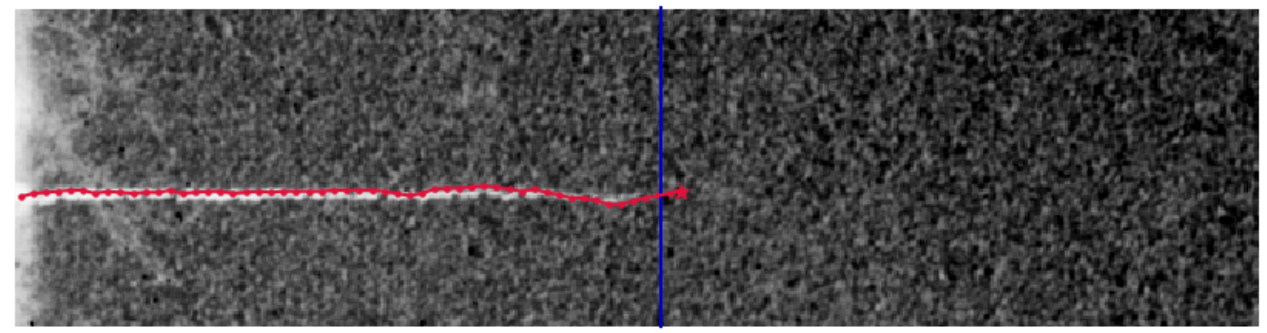

650

800
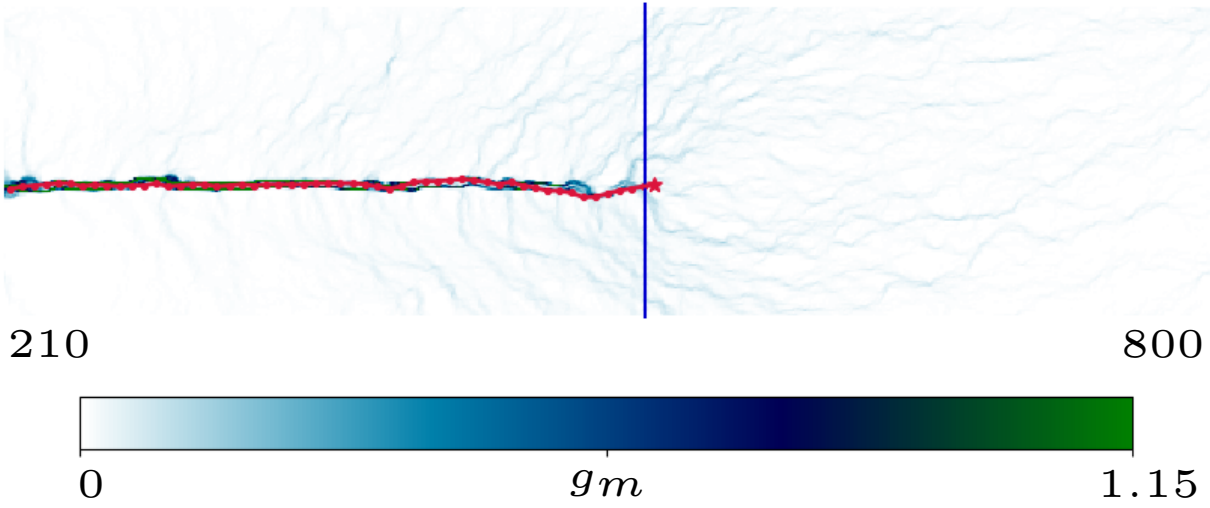

FIGURE 19 Result of the methodology using the final potential crack path for image 115 of Experiment 1 . The crack path (in red) is consistent with the crack visible in the inspection image and the gradient map. The horizontal position of the crack tip (marked with a red star) is close to the DCPD measurement (in blue).

too low evaluation for Threshold 1 on the absolute gradient of $\bar{\sigma}_{g m}$, i.e. a too low $\alpha$ coefficient. The first approximation of the crack tip is thus not correct for the corresponding images. This results in an incorrect evaluation for Threshold 2 on $\bar{\sigma}_{g m}$. For example, this is the case for image 175: The crack tip is then positioned too far along the potential crack path, as illustrated on Figure 20.

It is thus necessary to improve the evaluation of the $\alpha$ coefficient for Threshold 1 on the absolute gradient of $\bar{\sigma}_{g m}$ before considering this strategy to control an experiment based on the estimated crack length. However, let us emphasize that this example is difficult, as the speckle pattern is not very contrasted at high temperature.

\subsection{Analysis for Experiment 2 on the perforated specimen}

Figure 18 shows that for Experiment 2, the predicted crack tip position is in very good agreement with the one obtained with the DCPD method from image 16 up to image 100 for both the final crack path and the potential crack path on the current image. Before image 16, the crack propagation may not be stable for the results to be compared with the DCPD reference. From image 100, the presence of the hole influences the crack path and in the end, the plastic phenomena cannot be neglected and lead to a wide opening of the crack lips (see Figure 21). The difference between the crack length evaluations is then due to the overestimation of the crack length by the DCPD method. The crack length estimations provided by the proposed methodology are clearly more consistent than the DCPD method 


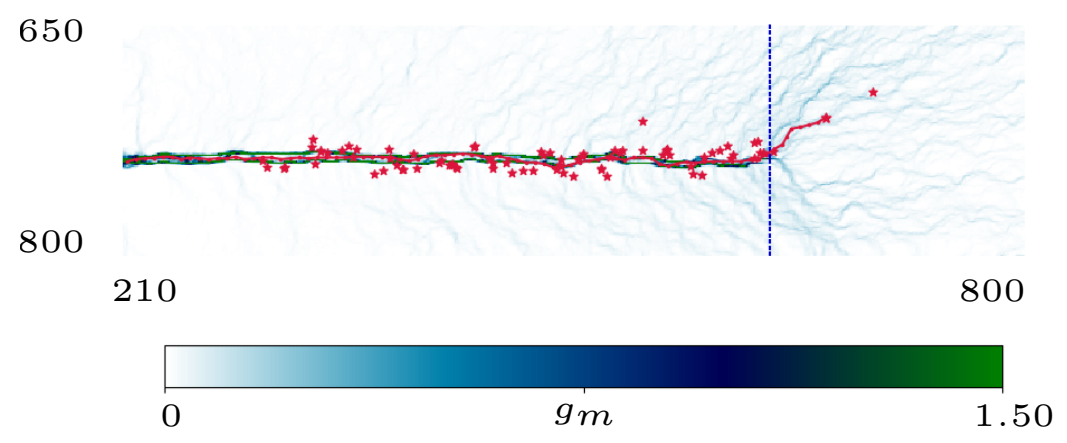

FIGURE 20 Maximum gradient map at image 175 with previous crack tips from image 13 (marked with red stars) for Experiment 1 at high temperature obtained with the potential crack path for the current image. The crack tips' positions are mostly consistent with the expected crack path. However, occasionally, using the potential crack path for the current image can lead to positioning the crack tip too far along the potential crack path.

ones with the maximum gradient maps and the visible blunt crack on Figure 21. 


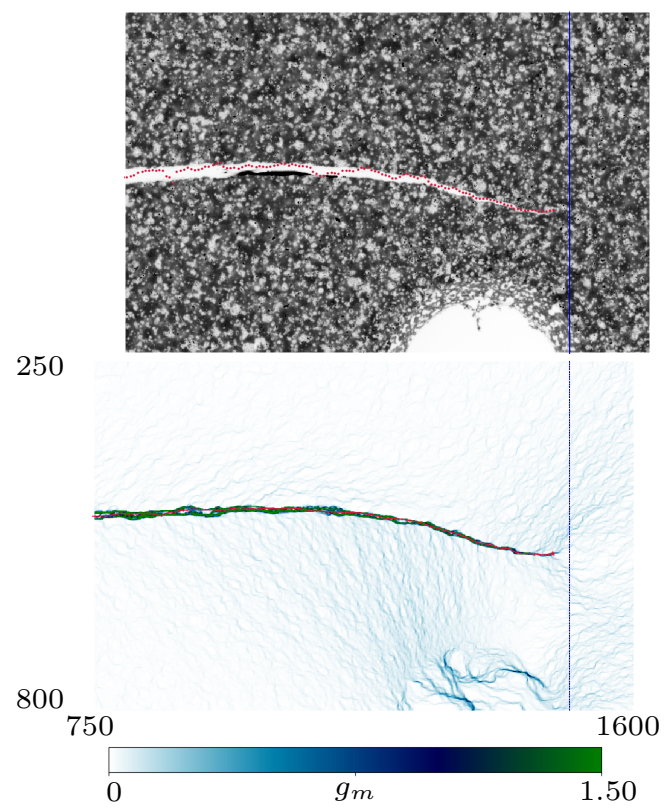

(a) Image 136

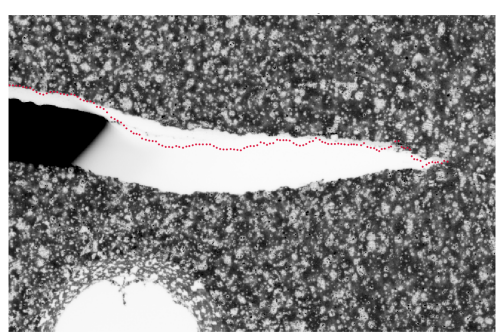

200

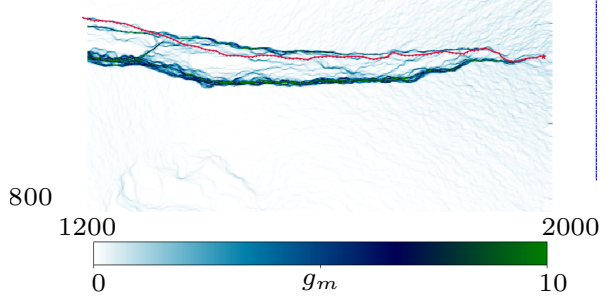

(b) Image 212

FIGURE 21 Crack paths (in red) obtained with the methodology using the final potential crack path for images 136 and 212 of Experiment 2. The crack paths are consistent with the crack lips positions near the crack tip and with the maximum gradient distribution visualized on the reference image. The crack tip horizontal position obtained thanks to the DCPD method is represented in blue: It is more and more overestimated between image 136 and image 212 . 


\subsection{Analysis on synthetic data based on Experiment 2}

A straight crack path approximation is considered here since it is assumed to be a reasonable assumption, at least at this stage of the propagation (see Figure 8). In this case, even if the actual crack path deviates from the straight assumed line, the crack tip detection criterion is still applicable since the absolute vertical deviation is less than half the window size. Threshold 1 already provides a satisfactory first estimation of the exact crack tip position with an error of about 6 pixels (in total or about 5.4 pixels horizontally, the vertical difference being set by the potential crack path choice in this case): See Figure 22. From here, the refined approximation obtained with Threshold 2 slightly diminishes the horizontal difference in the crack tip position to reach an error of about 4.7 pixels. Given the spatial resolution of the images, the final estimated crack tip is then at a distance of less than $100 \mu \mathrm{m}$. This slight error is deemed admissible: A perfect match cannot be expected, not only because the crack paths are not exactly coincident, but also because the displacement field used to deform the reference image is obtained from a finite element computation and is not an analytical one. We expect that the discretization may have an influence on the displacement field. Indeed, near the crack tip, the element size is about $80 \mu \mathrm{m}$, which is approximately equivalent to 4 pixels. This means that the strains supposed to be highly localized at the crack tip are actually rather distributed over that distance. Let us also underline that no a priori information about the loading or the constitutive material was used to obtain this result. This is why such a small difference (inferior to 10 pixels) validates the proposed criterion. 


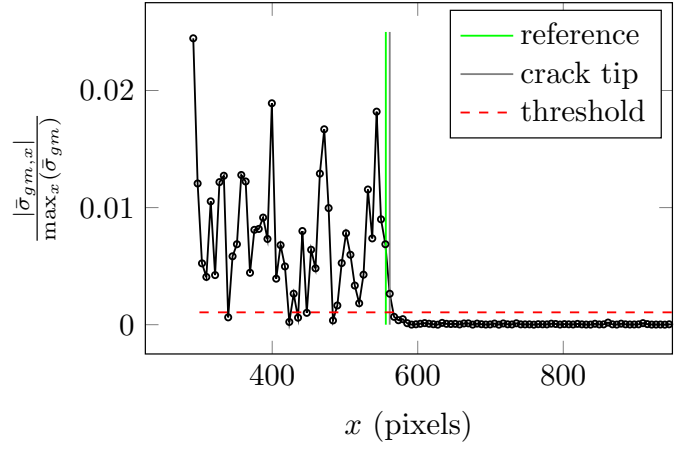

(a) Threshold 1 on $\left|\bar{\sigma}_{g m}, x\right|$.

0

1200

0

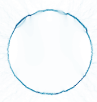

(c) First estimated crack tip position for Threshold 1 (in red) along the potential crack path (in gray)

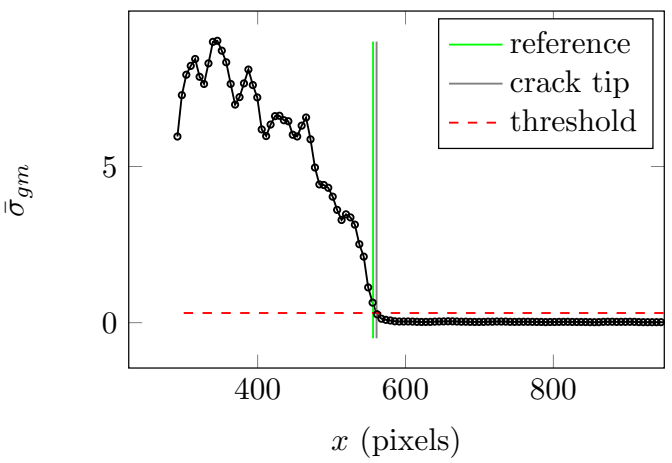

(b) Threshold 2 on $\bar{\sigma}_{g m}$.

430

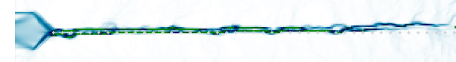

615 280

(d) Final estimated crack tip position for Threshold 2 (in red) zoomed in along the potential crack path (in gray)

FIGURE 22 Results for the synthetic data case based on Experiment 2. A first estimation of the crack tip position (gray line (a) or red dot (c)) is given by the intersection between $\left|\bar{\sigma}_{g m}, x\right|$ and Threshold 1 corresponding to $30 \%$ of its standard deviation. This first estimation corresponds already very well to the reference position (green line (b) or dot (d)). The estimation is then refined (gray line or red dot) thanks to the intersection of Threshold 2 with $\bar{\sigma}_{g m}$. In both cases, the estimated crack tip is less than 10 pixels away from the reference crack tip position, which validates the proposed crack tip detection criterion. 


\section{5 | CONCLUSION AND OUTLOOK}

In this paper, the authors propose a new methodology to determine the crack tip position from full field measurements independently of the loading conditions or the material constitutive behavior, which is also valid when large plastic deformations occur.

This methodology relies on the use of an optical flow estimation that provides overall smooth displacement fields except at the crack path, which corresponds to very localized high gradients and a potential crack path relatively aligned with this gradients.

To determine the crack tip position along a given potential crack path, the authors propose to rely on the drop of the normalized maximum gradient standard deviation $\bar{\sigma}_{g m}$. To begin with, the definition of a threshold of the absolute gradient of $\bar{\sigma}_{g m}$ leads to the determination of the last discrete point of the potential crack path at the transition between high oscillating values and a plateau of negligible gradients. This first estimation of the crack tip can then be refined by using another threshold for the normalized maximum gradient standard deviation $\bar{\sigma}_{g m}$. To define this second threshold, the gradients in the close vicinity of the crack tip are taken into account which distinguishes the proposed methodology from previous works.

The validity of the proposed methodology is verified by comparing of the position of the corresponding crack tip with the one obtained with the DCPD method, or with the visible crack tip on the inspection image when it is the only possible reference, on two fatigue crack growth experiments. The first experiment illustrates the robustness of the method for a globally straight crack propagation with load frequency changes at high temperature. The second experiment demonstrates the ability of the methodology to capture crack paths that are curved and to provide satisfactory results even when the DCPD method is biased, in particular when large deformations occur.

Since defining a crack tip location using only experimental results is quite challenging and the DCPD method is only valid under certain conditions, a validation case based on synthetic data comparable to one of the experiments is proposed. The crack tip location is retrieved with a difference of less than 10 pixels, which validates the proposed crack tip detection criterion.

Among the three main parameters introduced with the criterion, one parameter is essential to this methodology: The threshold on the absolute gradient of $\bar{\sigma}_{g m}$ used to determine the first crack tip estimation. Its determination could be improved by studying its optimal value for more cases. It would be particularly interesting to apply the same methodology for other materials such as concrete or composites, starting with the exact set of parameters selected in this study. This choice intervenes particularly when the crack path is determined in the currently studied image by the Marching Ridges algorithm using only the gradient distribution obtained with the DeepFlow measurement. The potential crack path then follows high gradient branches that are not entirely understood yet. More studies at the grain scale would be needed between the maximum and minimum loading stages to better understand the nature of the observed gradient branches, their evolution, and how they can affect crack propagation. However, if the proposed criterion is applied on the final crack path for every image, for the considered cases, the influence of this threshold value is reduced. With additional measures to detect punctual aberrant estimations, this algorithm could be used to provide more accurate results than the DCPD method for non straight cracks or in case of large plastic deformations for real-time experiment control.

In this paper, for the sake of brevity, only one optical flow algorithm has been considered. However, other optical flow algorithms that provide overall smooth displacement fields, except at the crack path where they must capture very localized high gradients, could be considered.

Furthermore, the potential of the Marching Ridges algorithm has not been fully exploited yet. Given its abilities 
for Finite Element computations, it should also be possible to apply this methodology to Digital Volume Correlation to capture three-dimensional crack paths or crack branching.

\section{Acknowledgements}

The experiments in this work were performed within the SEMAFOR project funded by the French Agence Nationale de la Recherche (ANR-14-CE07-0037 grant) for Experiment 1 at high temperature, and the GDR 3651 FataCrack framework (http://www.gdr3651.cnrs.fr/) for Experiment 2 on the perforated specimen. The methodology was developed within the framework of the PRF MECHANICS, an Onera-funded project. The images for the analysis based on synthetic data were provided by Max Sonzogni, Pascal Bouda, Thomas Fourest and Bertrand Langrand.

\section{A | FULL-FIELD MEASUREMENT}

\section{A.1 | Total Variation-like optical flow estimation}

Let us note an image $I$ as an intensity distribution $I(\underline{\mathbf{x}})>0$ defined for $\underline{\mathbf{x}}=[x, y]^{\mathrm{t}} \in \mathcal{S} \subset \mathbb{R}$. The general problem at hand is the estimation of a vector field $\underline{\mathbf{u}}(\underline{\mathbf{x}})=[u(\underline{\mathbf{x}}), v(\underline{\mathbf{x}})]^{t}$, also called the optical flow (OF), to map some inspection image $I$ onto a reference image, or template image, $T$, i.e.

$$
I(\underline{\mathbf{x}}) \approx T(\underline{\mathbf{x}}+\underline{\mathbf{u}}(\underline{\mathbf{x}})), \quad \forall \underline{\mathbf{x}}=[x, y]^{\mathrm{t}} \in \mathcal{S}
$$

Note that to account for image noise and other potential source of discrepancies between measurements, only an approximate identity between image intensities is required.

Numerous OF estimation methods proposed in the Computer Vision domain are based on the seminal work of Horn and Schunck [16]. These approaches adopt a regularization framework where the OF is determined by the minimization of a compound criterion

$$
\mathcal{J}(\underline{\mathbf{u}})=\mathcal{J}_{\text {data }}(\underline{\mathbf{u}} ; T, I)+\lambda \mathcal{J}_{\text {smooth }}(\underline{\mathbf{u}}) .
$$

Here the data term measures the registration error between $T$ and $I$, while the second term, called the regularization term, enforces the smoothness of the estimated vector field, both terms being balanced by a regularization parameter $\lambda>0$. These terms can be written as follows:

$$
\begin{gathered}
\mathcal{J}_{\operatorname{data}}(\underline{\mathbf{u}} ; T, I)=\int_{\underline{\mathbf{x}}} \phi_{D}\left(\mid T(\underline{\mathbf{x}})-I\left(\underline{\mathbf{x}}+\left.\underline{\mathbf{u}}(\underline{\mathbf{x}})\right|^{2}\right) \mathrm{d} \underline{\mathbf{x}}\right. \\
\mathcal{J}_{\text {smooth }}(\underline{\mathbf{u}})=\int_{\underline{\mathbf{x}}} \phi_{R}\left(|\nabla u|^{2}+|\nabla v|^{2}\right) \mathrm{d} \underline{\mathbf{x}}
\end{gathered}
$$

where $\nabla=[\partial / \partial x, \partial / \partial y]$. In these integrals, $r \mapsto \phi_{D}\left(r^{2}\right)$ and $r \mapsto \phi_{R}\left(r^{2}\right)$ are penalty functions which determine the cost associated to some residual $r$. The residual is either a difference of image intensities in the registration term, or the norm of a gradient of the displacement field in the regularization term $\mathcal{J}_{\text {smooth }}$. While historical works on OF use quadratic penalty functions $\left(\phi_{D}\left(r^{2}\right)=\phi_{R}\left(r^{2}\right)=r^{2}\right)$, since the late 90's several authors have proposed to use 
so-called "robust" functions, with an increasing rate slower than the quadratic. In the data term, a robust penalty function aims at allowing image difference to take large value in some places, for instance because of occlusion or illumination change. In the regularization term, it permits local large gradient, hence preserving local discontinuities of the displacement. A popular choice is $\phi_{R}\left(r^{2}\right)=\sqrt{r^{2}+\epsilon^{2}}$ which leads, using small values $\epsilon<<1$, to a smooth approximation of the $L^{1}$ or Total Variation (TV) norm of the gradient in the smoothness or regularization term.

Such TV-regularized OF estimation methods are very attractive to estimate the displacement around a crack, as illustrated in Figure 23. The dense, local DIC method of [21] is used with two different subset sizes: $31 \times 31$ (left) and $7 \times 7$ (middle). These results illustrate that local methods are trapped in a trade-off between smoothness far from the crack and accuracy near to it. More precisely, the DIC method uses a large subset and thus provides a smooth result. However, it also leads to an oversmoothing of the crack. Inversely, using a small subset leads to a better localization of the crack, but also to large noise effects. On the contrary, the robust TV-like method of [20] is able to provide both smoothness everywhere and localized high gradient just at the crack path.
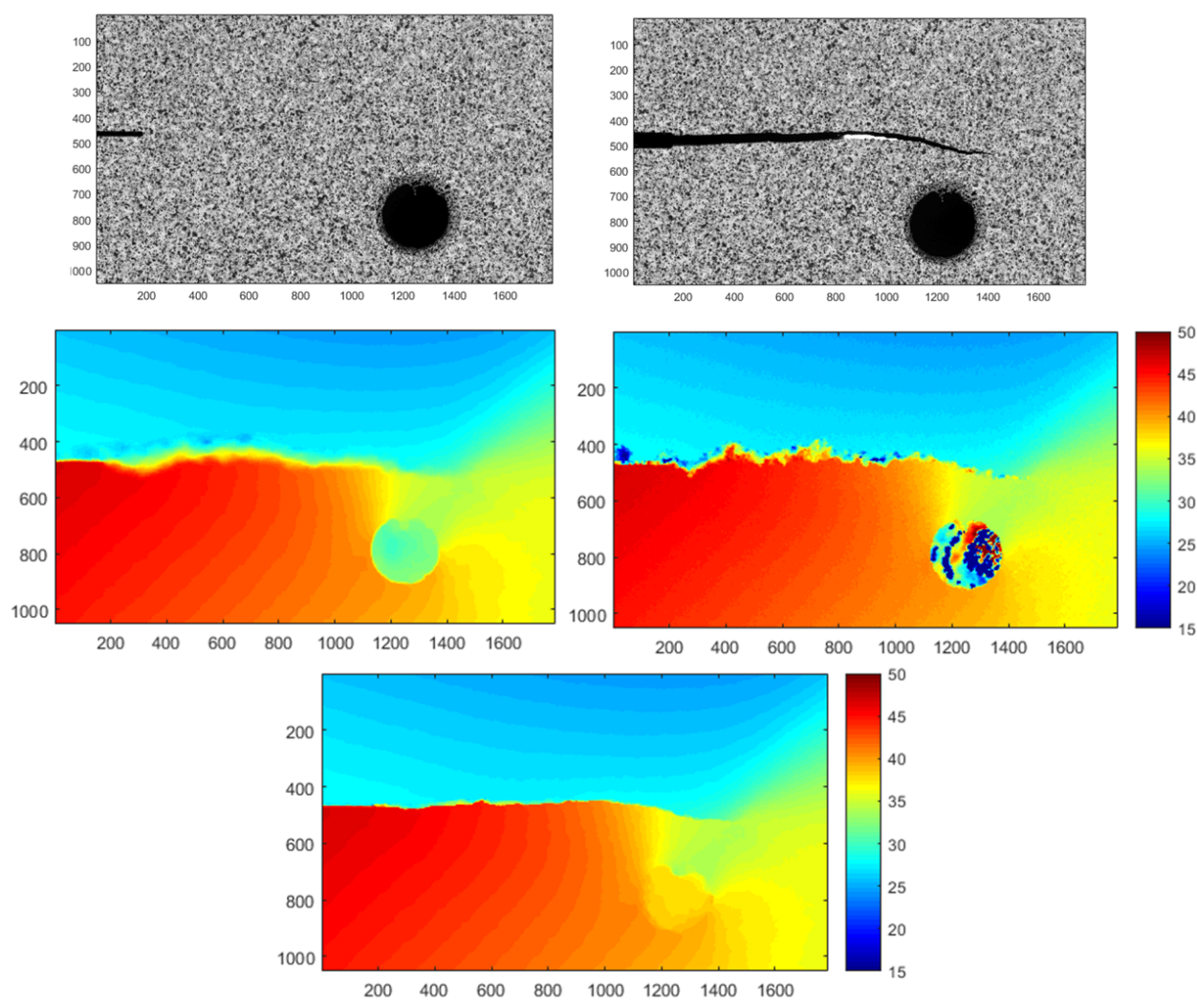

FIGURE 23 Comparison of local DIC method with TV regularization. Upper line: Two images from Experiment 2. Middle line: Norm of the displacement fields (in pixels) estimated with a local DIC method of [21] with a subset size of 31x31 pixels (left) and 7x7 pixels (right). Bottom line: Result of the OF algorithm of [38]. 
It is also interesting to look at the residual maps illustrated in Figure 24. A residual map is defined as

$$
\underline{\mathbf{x}} \mapsto \mathcal{E}(\underline{\mathbf{x}})=T(\underline{\mathbf{x}})-\tilde{I}(\underline{\mathbf{x}}+\underline{\hat{u}}(\underline{\mathbf{x}}))
$$

where $\tilde{I}$ denotes a bilinearly interpolated inspection image and $\underline{\hat{u}}$ is some estimated displacement field. In Figure 24 , the local DIC method with a $31 \times 31$ subset (top line) leads to high residual values in a large strip around the crack. Indeed, the estimated motion "slows down" as the subset is located closer to the crack, leading to high residuals. For a smaller subset (middle line), the crack is better resolved but high residuals due to noise occur. For the TV-like regularized flow of [38], the high residual are located only inside the crack support, meaning that the motion slows down only inside the crack (as the background visible through the crack does not move).

In this work, the DeepFlow algorithm [38] is used to provide all the displacement field estimations. No particular parameter specification is needed, probably due to the fact that this algorithm must be robust enough to be used in various contexts.
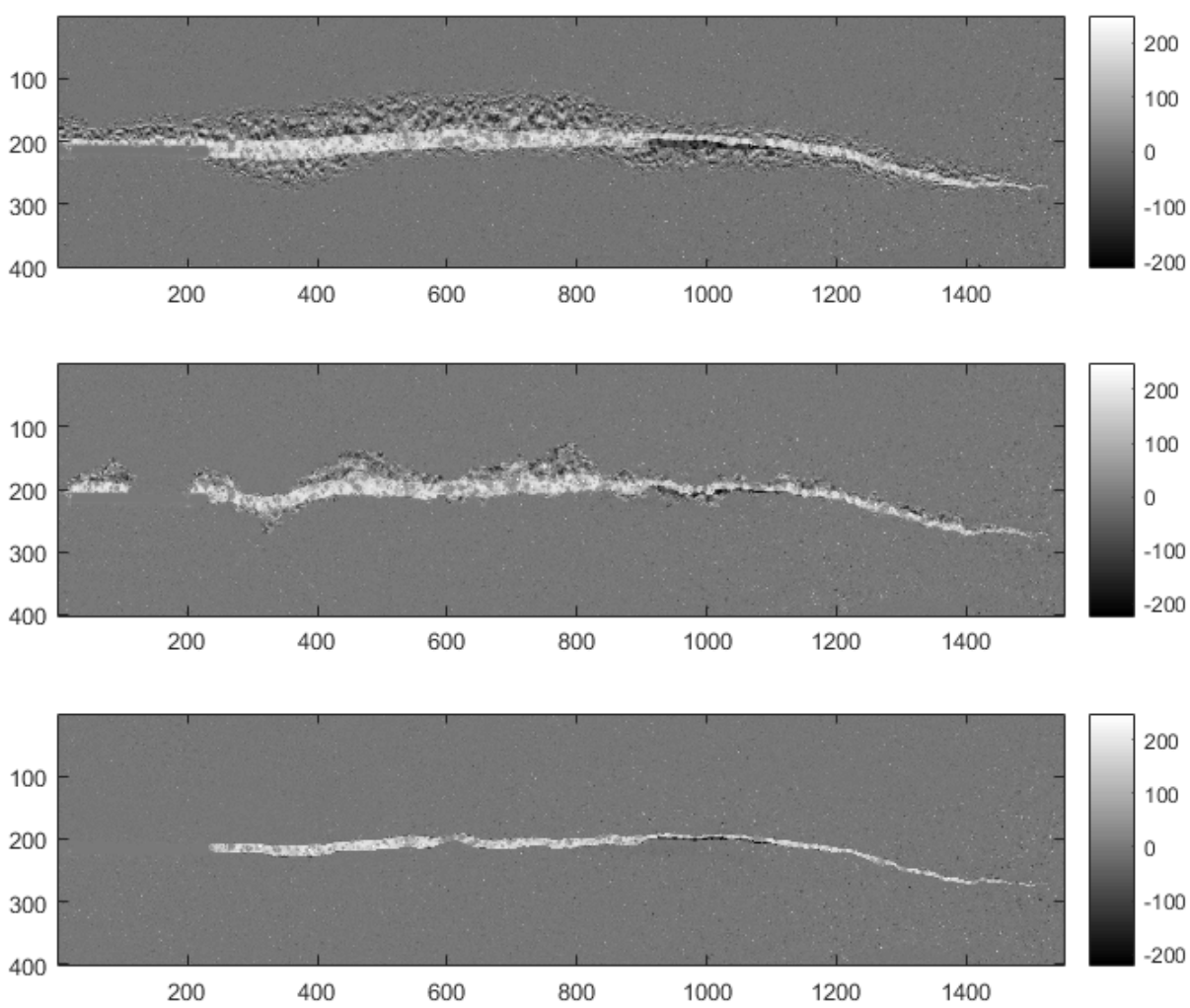

FIG URE 24 Residual maps deriving from the local DIC method of [21] with a subset size of 31x31 pixels (top) and of 7x7 pixels (middle). Bottom image: Residual map obtained with the OF algorithm of [38]. These residuals are measured in gray levels and the axes are in pixels. 


\section{A.2 | Dealing with very large displacements}

Optimization of (6) is computationally demanding and prone to convergence issues when very large displacements are sought for. The traditional way to improve the convergence is to use a Gaussian pyramid of images. In a pyramid with $L$ levels, the image on top (at level $L$ ) is obtained by low-pass filtering and undersampling the original image $L$ times, which means that its dimensions are $\left(M / 2^{L}, N / 2^{L}\right)$ if the original image is $(M, N)$. Large motions in the original image geometry are then divided by a factor $2^{L}$ in the highest level image. In a coarse-to-fine strategy, the displacement is initialized by zero at the highest level of the pyramid, then the criterion (6) is iteratively optimized between level $L$ images. The resulting OF is bilinearly expanded by a factor 2 , and used to initialized the level $L-1$ optimization, and so on until the original images have been registered.

Such a strategy is efficient, but can propagate errors when large motion of narrow objects is involved. More recently, some authors have proposed to initialize a TV-like algorithm with a first step of feature matching [5, 38]. Indeed very efficient and robust methods for feature matching have been proposed since the mid-2000's, including methods robust to large scale and rotation change, in keeping with [23]. In [5, 38], feature matching give "anchors" to the real displacement fields which are then used to initialize the optimization of criterion like (6), leading to better estimation of the large motions of small objects. However, such situations are not often encountered in the framework of full field measurement in material studies.

In this work, we use the version of the DeepFLow algorithm of [38] available in the OpenCV library (https://opencv.org/), which relies on a classical coarse-to-fine strategy on a Gaussian pyramid.

\section{B | POTENTIAL CRACK PATH TRACKING}

The first objective is to build, from the full field measurements, a discrete potential crack path. This path is constituted by segments of controlled size and corresponds to an approximation of the actual crack location on the DIC initial configuration (i.e. the undeformed configuration), followed by an approximation of the line where displacement gradients are the highest in front of the crack tip.

\section{B.1 | Marching ridges algorithm adaptation for full field measurements}

\section{B.1.1 | Principle}

Crack path tracking is performed based on the displacement field maps thanks to the Marching Ridges algorithm proposed by Feld-Payet et al. (see [10] for more details on the original algorithm). This algorithm was initially proposed and successfully applied to track crack paths from a scalar field related to material degradation resulting from a FE computation. Its principle is to approximate the ridge line of a scalar field viewed as a surface, by a set of contiguous segments.

In this work, for the first time, this algorithm is used to track crack paths based on full field measurements. The key point to this transposition is the choice of the new studied scalar field $f$. Indeed, this field must present a local extremum (maximum or minimum) at the crack lips. Obviously, this is not the case for the direct DIC output since none of the displacement field components does present an extremum value at the crack lips. However, the crack lips do correspond to a maximum of at least some components of the displacement gradient.

The choice of the precise gradient component is entirely related to the Marching Ridges strategy, specified here. 


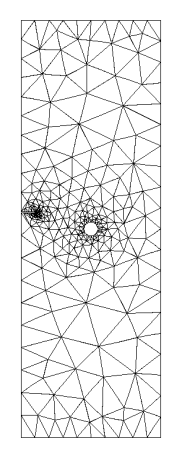

(a) Global mesh

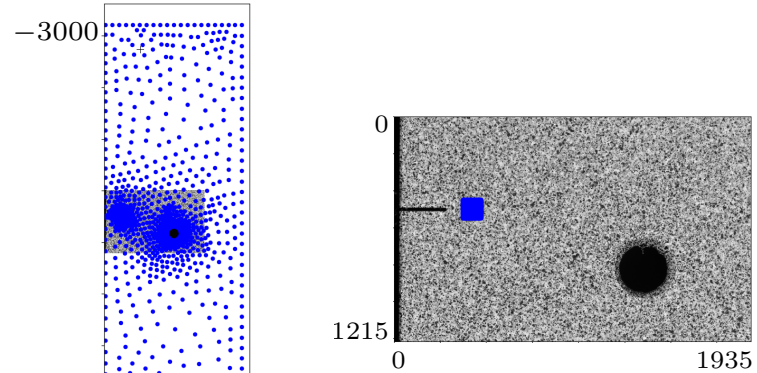

(c) Local mesh aligned with the reference image

(b) Global mesh aligned

with the reference image

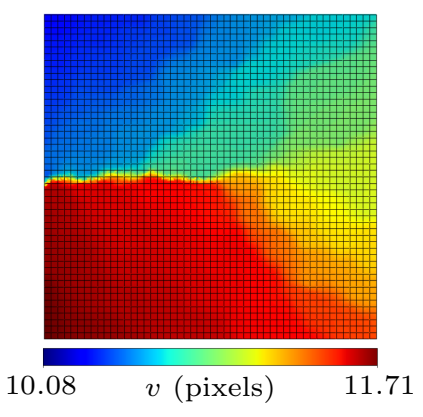

(d) Vertical displacement component transfered from the DIC pixel grid to the local mesh for image 8

FIGURE 25 After a first alignment of the specimen mesh with the reference image, the same homography transformation is applied to align the local mesh (of 50 by 50 elements) and be able to transfer the displacement components in the area of interest. In this case, one pixel corresponds to $37.2 \mu \mathrm{m}$, so each element of the local mesh is $74.4 \mu \mathrm{m}$ wide (while the whole specimen is $50 \mathrm{~mm}$ wide).

\section{B.1.2 | Practical strategy}

The steps of the considered Marching Ridges algorithm are quite standard for this type of algorithm (which was originally proposed in the Computer Vision domain, see e.g. [35] and [12]):

1. Initialization, i.e. specification of a starting point $\underline{\mathbf{x}}_{0}$ on the ridge;

2. Evaluation of the equations used to define a height ridge for several test transverse directions;

3. Expansion and marching, i.e. once a relative maximum is found, the same procedure is used from this new ridge point.

Besides, in this case, in order to use directly the FE implementation in the Zset code [3] of the Marching Ridges algorithm and to obtain the crack tip position in the real space (i.e. in millimeters), the displacement components are transferred onto a FE mesh.

\section{Transfer from a pixel grid to a finite element mesh}

To determine the correspondence between the pixel grid coordinates and the real space coordinates, the authors used the methodology described in [4] to find a homography transformation between the plane of the FE mesh of the whole specimen and the plane of the pixel grid. This step is performed once and for all with the reference image (which corresponds to the initial state of the unloaded specimen) and the same transformation is then used to transfer the displacements obtained by DIC (which are calculated on the reference image) onto a local rectangular mesh of smaller dimensions: See Figure 25 for an illustration on Experiment 2. The dimensions of the local mesh are chosen so that the distance between two nodes of the quadratic elements is one pixel. The displacement value at each node is then directly taken as equal to the value of the underneath pixel. 


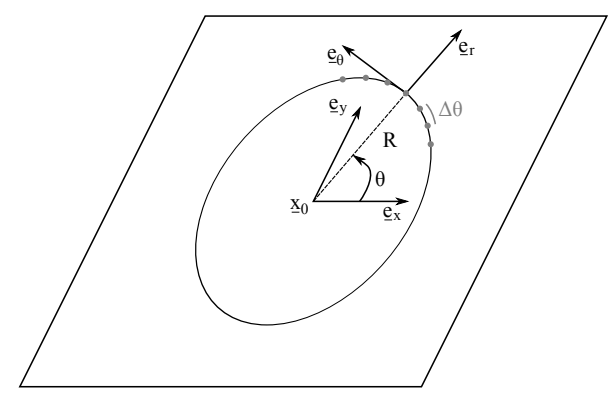

FIGURE 26 The polar grid centered at the considered point on the ridge is an original feature of the Marching Ridges algorithm proposed in [10]. Within this system, each tested point $\underline{\mathbf{x}}$, located at a distance with the center of the polar grid $R$, is positioned thanks to the angle $\theta$ with respect to the initial cartesian coordinate system. Each tested point can thus be associated with a couple of unit vectors $\left(\underline{\mathbf{e}}_{r}, \underline{\mathbf{e}}_{\theta}\right)$.

\section{Initialization}

Initialization of the search takes advantage of the fact that the considered specimens for fatigue crack propagation are pre-notched and the geometry of the notches is well known. The first crack tip is thus specified (in the real space) to correspond to the tip of the notch and the direction of the pre-notch is also taken into account in order to search the next front only ahead of the crack tip.

\section{Test of different directions}

One of the main original feature of this algorithm, is that the search for the ridge direction is performed on a polar coordinate system centered on a point of the ridge $\underline{\mathbf{x}}_{0}$ (whereas in Computer Vision, a cartesian coordinate system, which usually fits the grid of pixels, is generally used), as illustrated on Figure 26 . The idea is then to find the next point on the ridge at a user chosen distance $R$ with an angular precision equal to the grid angular step $\Delta \theta$. The main advantage of the angular step is that is allows the user to control the accuracy of the approximated ridge line while allowing the determined crack path to be less dependent on the discretization of the underlying grid.

For each tested direction $\theta$ (corresponding to a vector $\underline{\mathbf{e}}_{\theta}(r)$, the aim is to determine whether, according to a height ridge definition, there is a relative maximum of the studied function in the transverse direction $\underline{\mathbf{e}}_{\theta}(\theta)$. To do so, the authors of [10] proposed to study the change of sign of the scalar product of the considered function gradient with the orthoradial vector $\underline{\mathbf{e}}_{\theta}$. Indeed, the change of sign of this scalar product from positive to negative corresponds to a ridge line, as long as the local curvature radius of the considered field is smaller than the evaluation radius $R$. As far as the choice of the function of interest for full-field measurements is concerned, given the use of a polar grid, the authors here propose to consider the partial derivative of the displacement norm with respect to the angular parameter $\theta$, i.e. $\frac{\partial\|\underline{\underline{u}}\|}{\partial \theta}$, where $\|\cdot\|$ designates the euclidean norm. Consequently, for the angle $\theta$ to correspond with an angular precision of $\Delta \theta$ to the ridge orientation (assuming that the ridge line can be locally approximated by a straight line on a distance $R$ from the point $\underline{\mathbf{x}}$ ), a sufficient condition is:

$$
\begin{cases}\underline{\mathbf{e}}_{\theta}(\theta-\Delta \theta / 2) \cdot \nabla\left(\frac{\partial\|\underline{\underline{u}}\|}{\partial \theta}\right)\left(\underline{\mathbf{x}}+R \underline{\mathbf{e}}_{r}(\theta-\Delta \theta / 2)\right) & >0 \\ \underline{\mathbf{e}}_{\theta}(\theta+\Delta \theta / 2) \cdot \nabla\left(\frac{\partial \| \underline{\underline{u} \|}}{\partial \theta}\right)\left(\underline{\mathbf{x}}+R \underline{\mathbf{e}}_{r}(\theta+\Delta \theta / 2)\right) & <0\end{cases}
$$

What is actually studied is thus the change of sign of the second derivative of the displacement norm: $\frac{\partial^{2}\|\mathbf{u}\|}{\partial \theta^{2}}$. 
In practice, this quantity is simply approximated from the first derivative $\frac{\partial\|\underline{u}\|}{\partial \theta}=R \underline{\mathbf{e}}_{\theta} \cdot \underline{\nabla}\|\underline{\mathbf{u}}\|$ thanks to a finite difference (which is valid as long as the angular accuracy $\Delta \theta$ remains small). The final set of equations corresponding to the ridge detection is then:

$$
\begin{cases}\frac{\partial^{2}\|\underline{\underline{u}}\|}{\partial \theta^{2}}(\theta-\Delta \theta / 2) \approx \frac{\frac{\partial\|\underline{\underline{u}}\|}{\partial \theta}(\theta-\Delta \theta / 2)-\frac{\partial\|\| \underline{u} \|}{\partial \theta}(\theta-2 \Delta \theta / 2)}{\Delta \theta} & >0 \\ \frac{\partial^{2}\|\underline{\underline{u}}\|}{\partial \theta^{2}}(\theta+\Delta \theta / 2) \approx \frac{\frac{\partial\|\underline{\underline{u}}\|}{\partial \theta}(\theta+\Delta \theta / 2)-\frac{\partial\|\underline{\underline{u}}\|}{\partial \theta}(\theta-\Delta \theta / 2)}{\Delta \theta} & <0\end{cases}
$$

Besides, in order to reduce the effect of the evaluation radius choice and thus bring to the methodology more robustness, the sign of the scalar products in the equation set 11 is computed on several radii $R_{i}$ around the main evaluation radius $\mathrm{R}$, and what is considered is the mean first derivative:

$$
\frac{\partial\|\underline{\mathbf{u}}\|}{\partial \theta}_{\mid \text {mean }}(\theta)=R \underline{\mathbf{e}}_{\theta}(\theta) \cdot\left[\sum_{i=0}^{n-1} \underline{\nabla}\|\underline{\mathbf{u}}\|\left(\underline{\mathbf{x}}_{0}+R_{i} \underline{\mathbf{e}}_{r}(\theta)\right)\right]
$$

where $R_{i}=R-\frac{L}{2}+\frac{i L}{n-1}$ designates the $n$ evaluation radii, $n$ being set arbitrarily to 10 and $L$ being the width of the evaluation band, taken 3.5 times larger than the distance between two nodes.

As the previous set of equation 11 can be verified for several angles $\theta$, it is possible to detect simultaneously several ridge directions. However, in this paper, only the direction corresponding to the largest mean gradient $\frac{\partial\|\underline{\mathbf{u}}\|}{\partial \theta}{ }_{\mid \text {mean }}$ ahead of the crack tip is chosen since there is only one crack branch to follow.

\section{Expansion and marching}

Within the same image After a new increment is found, the same search is resumed from the end of the last increment until it is no longer possible to find a direction $\theta$ verifying the set of equations 12 or because the corresponding mean gradient value is too low, i.e. less than a user defined threshold.

Contrary to the Finite Element computation framework where the crack advance corresponds to a rather small length with respect to the whole specimen mesh, for full-field measurement analysis, the crack advance may easily exceed the boundary of the local mesh. In that case, at the end of a search on a local mesh, the found crack tip is very close to a boundary. To preserve as much accuracy as possible with the local mesh while keeping computational costs reasonable, the authors have chosen to move the mesh at the end of each search (rather than to choose a larger mesh) so that the mesh center corresponds to the last found crack tip position. When the crack tip position has not changed between two mesh changes, the authors consider that the final crack tip position for the image has been found.

Let us note that the presence of a boundary may interfere with the crack path search due to the lack of data. In order to overcome this issue and bring more robustness to the strategy, at each mesh change, the search is resumed from a few increments back. For example, when the crack increment length is equal to 4 times the size of an element, it would take at least 12 increments to cross a local mesh of 50 by 50 elements so the search is resumed 2 to 3 increments back.

Between two images The same principle is applied between two images. It may be particularly helpful to resume from a few steps back if there was a correlation problem near the crack tip for one image, but there is no problem on the next image in this area. It can also be helpful to take into account crack path changes after unloading and reloading because of changes at the grain scale. 
This incremental crack path construction between two (or more) images, which is quite similar to the one used for FE computations, ensures that crack propagation can be followed even if the crack lips are far apart at a certain distance from the crack tip where the crack path has already been determined.

\section{B.1.3 | Marching Ridges parameters}

The Marching Ridges algorithm involves the choice of two main parameters, the angular accuracy $\Delta \theta$ and the crack increment length $d L$, for which the authors provide some guidance here.

\section{Angular accuracy}

Because of the sparse nature of the available data, the choice of the angular accuracy and the choice of the evaluation radius $R$ are actually strongly related. Indeed, for a given angular accuracy $\Delta \theta$, the associated evaluation radius should be of the order ${ }^{3}$ of $\frac{1}{\tan (\Delta \theta)}$, i.e. for an angular accuracy between 5 and 10 degrees, the evaluation radius should be between 5 to 12 pixels. So choosing a too small angular accuracy would lead to a too large evaluation radius and a more averaged estimation of the crack path. Besides, it would also lead to an increase of the computational costs since there would be more evaluations of 10 . On the contrary, choosing values greater than 10 degrees could lead to a poorer (in particular less smooth) estimation of the actual crack path. Besides, for the approximation of the second gradient in the set of equations 11 to be accurate, it requires the angular accuracy to be small enough. For the angular accuracy choice, to provide a sufficiently accurate approximation of the crack path, the authors recommend values between 5 to 10 degrees. In this paper, the angular accuracy is set to 5 degrees.

\section{Crack increment length}

The radius of evaluation $R$ being fixed by the angular accuracy, another parameter is introduced to allow insertion of crack increments that are shorter than $R$ : The crack increment length $d L$. Its choice is a compromise. On the one hand, a small crack increment allows to :

- Capture large crack path curvatures;

- Be small regarding the size of the local mesh to be able to remove some increments between two local meshes or two images ${ }^{4}$;

On the other hand, it also:

- Increases the global computational costs since there would be more steps of ridge direction evaluation.

- Should not be smaller than one element of the local mesh. That is why the authors recommend to define the crack length in terms of multiples of the local mesh element size (which corresponds here to 2 pixels).

In this paper, the crack increments are equal to 3 times the local mesh element size (i.e. $3 \times 2=6$ pixels). Such a fine level of crack path representation may not be needed to represent a crack for a Finite Element computation, but it is

\footnotetext{
${ }^{3}$ More precisely, an approximation of the evaluation radius can be directly computed from the angular accuracy $\Delta \theta$ and the distance corresponding to the grid step $\Delta /$ thanks to the following formula: $R=\frac{\Delta l}{\tan (\Delta \theta)}$.

${ }^{4}$ On the contrary, Choosing a larger crack increment length could lead to increase the local mesh size and thus the transfer related computational cost for a constant element size
} 
necessary to properly capture the crack path for Experiment 1 , since it is, at the grain scale, far from straight.

\section{references}

[1] ASTM E647. Standard Test Method for Measurement of Fatigue Crack Growth Rates, ASTM International; 2015.

[2] Bouda P., Langrand B., Notta-Cuvier D., Markiewicz E., Pierron F. A computational approach to design new tests for viscoplasticity characterization at high strain-rates. Computational Mechanics, 2019.

[3] Besson J, Foerch R. Large scale object-oriented finite element code design. Computer Methods in Applied Mechanics Engineering 1997;142:165-187.

[4] Bettonte F. Development of a parameter identification strategy using Finite Element Model Updating on Digital Image Correlation measurements: towards the application to a non-local damage model. PhD thesis, PSL Research University; 2017.

[5] Brox T, Bregler C, Malik J. Large displacement optical flow. In Computer Vision and Pattern Recognition (CVPR) 2009;p. 41-48.

[6] Brox T, Bruhn A, Papenberg N, Weickert J. High accuracy optical flow estimation based on a theory for warping. ECCV 2004;p. 25-36.

[7] Doremus L. Calibration of the potential drop method for monitoring small crack growth from surface anomalies - crack front marking technique and finite element simulations. Int J Fatigue 2015;70:178-185.

[8] Durif E, Réthoré J, Combescure A, Fregonese M, Chaudet P. Controlling stress intensity factors during a fatigue crack propagation using digital image correlation and a load shedding procedure. Experimental Mechanics 2011;52:1021-1031.

[9] Fagerholt E, Børvik T, Hopperstad O. Measuring discontinuous displacement fields in cracked specimens using digital image correlation with mesh adaptation and crack-path optimization. Optics and Lasers in Engineering 2013;51(3):299310.

[10] Feld-Payet S, Chiaruttini V, Besson J, Feyel F. A new marching ridges algorithm for crack path tracking in regularized media. International Journal of Solids and Structures 2015;(71):57-69.

[11] Fessler E, Andrieu E, Bonnand V, Chiaruttini V, Pierret S. Relation between crack growth behaviour and crack front morphology under hold-time conditions in DA Inconel 718. International Journal of Fatigue 2017;96:17 - 27.

[12] Furst JD, Pizer SM. Marching ridges. In: SIP; 2001. p. 22-26.

[13] Grégoire D. Initiation, propagation, arrêt et redémarrage de fissures sous impact. PhD thesis, Insa Lyon; 2008.

[14] Hamam R, Hild F, Roux S. Stress intensity factor gauging by digital image correlation: application in cyclic fatigue. Strain 2007;(43):181-192.

[15] Henninger C, Hild F, Roux S. Enriched kinematic fields of cracked structures. International Journal of Solids and Structures 2010;(47):3305-3316.

[16] Horn BK, Schunck BG. Determining optical flow. Artificial intelligence 1981;17(1-3):185-203.

[17] Hos Y, Freire JLF, Vormwald M. Measurements of strain fields around crack tips under proportional and non-proportional mixed-mode fatigue loading. International Journal of Fatigue 2016;(89):87-98.

[18] Hosdez J, Witz JF, Martel C, Limodin N, Najjar D, Charkaluk E, et al. Fatigue crack growth law identification by Digital Image Correlation and electrical potential method for ductile cast iron. Engineering Fracture Mechanics 2017;182:577 $-594$. 
[19] Johnson HH. Calibrating the electrical potential method for studying slow crack growth. Mater Res Standard 1965;5(9):442-445.

[20] Le Besnerais G, Champagnat F. B-spline image model for energy minimization-based optical flow estimation. IEEE Trans on Image Processing 2006;(15):3201-3206.

[21] Le Besnerais G, Le Sant Y, Lévêque D. Fast and dense 2D and 3D displacement field estimation by a highly parallel image correlation algorithm. Strain 2016;52(4):286-306.

[22] Leplay P, Réthoré J, Meille S, Baietto MC. Identification of damage and cracking behaviours based on energy dissipation mode analysis in a quasi-brittle material using digital image correlation. International Journal of Fracture 2011;(171):3550 .

[23] Lowe DG. Object recognition from local scale-invariant features. In: Computer vision, 1999. The proceedings of the seventh IEEE international conference on, vol. 2 leee; 1999. p. 1150-1157.

[24] Hicks MA and Pickard AC. A comparison of theoretical and experimental methods of calibrating the electrical potential drop technique for crack length determination. International Journal of Fracture 1982;20:91-101.

[25] Mohan A, Poobal S. Crack detection using image processing: A critical review and analysis. Alexandria Engineering Journal 2017;

[26] Mokhtarishirazabad M, Lopez-Crespo P, B M, Lopez-Moreno A, Zanganeh M. Evaluation of crack-tip fields from DIC data: A parametric studyigue loading. International Journal of Fatigue 2016;(89):11-19.

[27] Paris P, Erdogan F. A critical analysis of crack propagation laws. J Basic Eng 1962;85:528-533.

[28] Rice JR. A path independent integral and the approximate analysis of strain concentration by notches and cracks. Journal of Applied Mechanics 1968;35:379--386.

[29] Ritchie RO, Bathe KJ. On the calibration of the electrical Potential Technique for monitoring crack growth using Finite Element Methods. International Journal of Fracture 1979;15:47-55.

[30] Roux S, Réthoré J, Hild F. Digital Image Correlation and Fracture: An Advanced Technique for Estimating Stress Intensity Factors of 2D and 3D Cracks. Journal of Physics D: Applied Physics 2009;42:214004 (21pp).

[31] Réthoré J. Automatic crack tip detection and stress intensity factors estimation of curved cracks from digital images. Int J Numer Meth Engng 2015;(103):516-534.

[32] Réthoré J, Estevez R. Identification of a cohesive zone model from digital images at the micron-scale. Journal of the Mechanics and Physics of Solids 2013;61:1407-1420.

[33] Réthoré J, Hild F, Roux S. Extended digital image correlation with crack shape optimization. Int J Numer Meth Engng 2008;(73):248-272.

[34] Sinha SK, Fieguth PW. Automated detection of cracks in buried concrete pipe images. Automation in construction 2006;15:58-72.

[35] Thirion JP, Gourdon A. The Marching lines algorithm : new results and proofs; 1993.

[36] Tong, J. Full-field characterisation of crack tip deformation and fatigue crack growth using digital image correlation-a review Fatigue and Fracture of Engineering Materials and Structures 2018;(41):1855-1869

[37] Turner D. Z. Peridynamics-Based Digital Image Correlation Algorithm Suitable for Cracks and Other Discontinuities Journal of Engineering Mechanics 2015; (141):04014115 
[38] Weinzaepfel P, Revaud J, Harchaoui Z, Schmid C. DeepFlow: Large displacement optical flow with deep matching. ICCV 2013;.

[39] Wells AA. The condition of fast fracture in aluminium alloys with particular reference to comet failures. Technical report of British Welding Research Association 1955;.

[40] Yadav PS, Patil J, Patil P, Lahane V, Patil H. Crack detectionh using image processing: review. International Journal of Engineering Research and Technology 2018;7.

[41] Yoneyama S, Morimoto Y, Takashi M. Automatic evaluation of mixed-mode stress intensity factors utilizing digital image correlation. Strain 2006;(42):21-29. 